GLASNIK MATEMATIČKI

Vol. 39(59)(2004), $155-170$

\title{
ON RECTANGULAR INVERSE SYSTEMS OF TOPOLOGICAL SPACES
}

\author{
Sibe MARDEŠIĆ \\ University of Zagreb, Croatia
}

\begin{abstract}
For every cofinite inverse system of compact Hausdorff spaces $\boldsymbol{X}=\left(X_{\lambda}, p_{\lambda \lambda^{\prime}}, \Lambda\right)$, there exists a cofinite inverse system of compact polyhedra $\boldsymbol{Z}=\left(Z_{\lambda}^{\tau}, r_{\lambda \lambda^{\prime}}^{\tau \tau^{\prime}}, \Lambda \times T\right)$ and there are mappings $u_{\lambda}^{\tau}: X_{\lambda} \rightarrow Z_{\lambda}^{\tau}$, $(\lambda, \tau) \in \Lambda \times T$, such that $u_{\lambda}^{\tau} p_{\lambda \lambda^{\prime}}=r_{\lambda \lambda^{\prime}}^{\tau \tau} u_{\lambda^{\prime}}^{\tau}$, for $\lambda \leq \lambda^{\prime}$, and $r_{\lambda}^{\tau \tau^{\prime}} u_{\lambda}^{\tau^{\prime}}=$ $u_{\lambda}^{\tau}$, for $\tau \leq \tau^{\prime}$. Moreover, for every $\lambda \in \Lambda$, the mapping $\boldsymbol{u}_{\lambda}: X_{\lambda} \rightarrow$ $\boldsymbol{Z}_{\lambda}=\left(Z_{\lambda}^{\tau}, r_{\lambda \lambda}^{\tau \tau^{\prime}}, T\right)$, given by the mappings $u_{\lambda}^{\tau}, \tau \in T$, is a limit of $\boldsymbol{Z}_{\lambda}$. If mappings $p_{\lambda}: X \rightarrow X_{\lambda}$ form a limit $\boldsymbol{p}: X \rightarrow \boldsymbol{X}$, then the mappings $v_{\lambda}^{\tau}=u_{\lambda}^{\tau} p_{\lambda}: X \rightarrow Z_{\lambda}^{\tau}$ form a limit $\boldsymbol{v}: X \rightarrow \boldsymbol{Z}$. An analogous result holds for cofinite inverse systems of topological spaces and ANR-resolutions (polyhedral resolutions).
\end{abstract}

\section{INTRODUCTION}

By a rectangular or double inverse system we mean an inverse system $\boldsymbol{Z}=\left(Z_{\nu}, r_{\nu \nu^{\prime}}, N\right)$, whose index set $N=\Lambda \times T$ is the product of two directed sets $\Lambda$ and $T$. By definition, $\Lambda \times T$ is ordered by the product ordering, i.e., $(\lambda, \tau) \leq\left(\lambda^{\prime}, \tau^{\prime}\right)$, provided $\lambda \leq \lambda^{\prime}$ and $\tau \leq \tau^{\prime}$. If $\nu=(\lambda, \tau) \in \Lambda \times T$, we denote $Z_{\nu}=Z_{(\lambda, \tau)}$ by $Z_{\lambda}^{\tau}$. Similarly, if $\nu=(\lambda, \tau) \leq\left(\lambda^{\prime}, \tau^{\prime}\right)=\nu^{\prime}$, we denote $r_{\nu \nu^{\prime}}=r_{(\lambda, \tau)\left(\lambda^{\prime}, \tau^{\prime}\right)}$ by $r_{\lambda \lambda^{\prime}}^{\tau \tau^{\prime}}$. If $\tau=\tau^{\prime}$ or $\lambda=\lambda^{\prime}$, we use shorter notation $r_{\lambda \lambda^{\prime}}^{\tau}$ and $r_{\lambda}^{\tau \tau^{\prime}}$, respectively. We will refer to $r_{\lambda \lambda^{\prime}}^{\tau}$ and $r_{\lambda}^{\tau \tau^{\prime}}$ as to horizontal and vertical bonding mappings of $\boldsymbol{Z}$, respectively. Clearly, if $(\lambda, \tau) \leq\left(\lambda^{\prime}, \tau^{\prime}\right)$, then $(\lambda, \tau) \leq\left(\lambda^{\prime}, \tau\right) \leq\left(\lambda^{\prime}, \tau^{\prime}\right)$ and $(\lambda, \tau) \leq\left(\lambda, \tau^{\prime}\right) \leq\left(\lambda^{\prime}, \tau^{\prime}\right)$. Consequently,

$$
r_{\lambda \lambda^{\prime}}^{\tau} r_{\lambda^{\prime}}^{\tau \tau^{\prime}}=r_{\lambda \lambda^{\prime}}^{\tau \tau^{\prime}}=r_{\lambda}^{\tau \tau^{\prime}} r_{\lambda \lambda^{\prime}}^{\tau^{\prime}}
$$

2000 Mathematics Subject Classification. 54B35.

Key words and phrases. Inverse system, rectangular inverse system, inverse limit, iterated inverse limit, compact Hausdorff space, compact metric space, polyhedron, ANR. 
which shows that the horizontal and the vertical bonding mappings determine all bonding mappings in a rectangular system. For any fixed $\lambda \in \Lambda, Z_{\lambda}=$ $\left(Z_{\lambda}^{\tau}, r_{\lambda}^{\tau \tau^{\prime}}, T\right)$ is an inverse system, called the vertical system of $\boldsymbol{Z}$ at level $\lambda$. The mappings $r_{\lambda \lambda^{\prime}}^{\tau}: Z_{\lambda^{\prime}}^{\tau} \rightarrow Z_{\lambda}^{\tau}, \tau \in T$, form a level mapping $\boldsymbol{r}_{\lambda \lambda^{\prime}}: \boldsymbol{Z}_{\lambda^{\prime}} \rightarrow \boldsymbol{Z}_{\lambda}$. For $\lambda \leq \lambda^{\prime} \leq \lambda^{\prime \prime}, \boldsymbol{r}_{\lambda \lambda^{\prime}} \boldsymbol{r}_{\lambda^{\prime} \lambda^{\prime \prime}}=\boldsymbol{r}_{\lambda \lambda^{\prime \prime}}$. Similarly, for any fixed $\tau \in T, \boldsymbol{Z}^{\tau}=$ $\left(Z_{\lambda}^{\tau}, r_{\lambda \lambda^{\prime}}^{\tau}, \Lambda\right)$ is an inverse system, called the horizontal system of $\boldsymbol{Z}$ at level $\tau$. The mappings $r_{\lambda}^{\tau \tau^{\prime}}: Z_{\lambda}^{\tau^{\prime}} \rightarrow Z_{\lambda}^{\tau}, \lambda \in \Lambda$, form a level mapping $\boldsymbol{r}^{\tau \tau^{\prime}}: \boldsymbol{Z}^{\tau^{\prime}} \rightarrow \boldsymbol{Z}^{\tau}$. For $\tau \leq \tau^{\prime} \leq \tau^{\prime \prime}, \boldsymbol{r}^{\tau \tau^{\prime}} \boldsymbol{r}^{\tau^{\prime} \tau^{\prime \prime}}=\boldsymbol{r}^{\tau \tau^{\prime \prime}}$.

If $\boldsymbol{X}=\left(X_{\lambda}, p_{\lambda \lambda^{\prime}}, \Lambda\right)$ is an inverse system of spaces and $u_{\lambda}^{\tau}: X_{\lambda} \rightarrow Z_{\lambda}^{\tau}$ are mappings such that

$$
u_{\lambda}^{\tau} p_{\lambda \lambda^{\prime}}=r_{\lambda \lambda^{\prime}}^{\tau} u_{\lambda^{\prime}}^{\tau}, \lambda \leq \lambda^{\prime},
$$

then the mappings $u_{\lambda}^{\tau}, \lambda \in \Lambda$, form a level mapping $\boldsymbol{u}^{\tau}: \boldsymbol{X} \rightarrow \boldsymbol{Z}^{\tau}$. If

$$
r_{\lambda}^{\tau \tau^{\prime}} u_{\lambda}^{\tau^{\prime}}=u_{\lambda}^{\tau}, \tau \leq \tau^{\prime}
$$

then the mappings $u_{\lambda}^{\tau}: X_{\lambda} \rightarrow Z_{\lambda}^{\tau}, \tau \in T$, form a mapping $\boldsymbol{u}_{\lambda}: X_{\lambda} \rightarrow \boldsymbol{Z}_{\lambda}$. If both (1.2) and (1.3) hold, then $\boldsymbol{u}_{\lambda} p_{\lambda \lambda^{\prime}}=\boldsymbol{r}_{\lambda \lambda^{\prime}} \boldsymbol{u}_{\lambda^{\prime}}$ and $\boldsymbol{r}^{\tau \tau^{\prime}} \boldsymbol{u}^{\tau^{\prime}}=\boldsymbol{r}^{\tau}$.

Assuming (1.2) and (1.3), every mapping $\boldsymbol{p}: X \rightarrow \boldsymbol{X}$, which consists of mappings $p_{\lambda}: X \rightarrow X_{\lambda}$, induces a mapping $\boldsymbol{v}: X \rightarrow \boldsymbol{Z}$, which consists of mappings $v_{\lambda}^{\tau}: X \rightarrow Z_{\lambda}^{\tau}$, where

$$
v_{\lambda}^{\tau}=u_{\lambda}^{\tau} p_{\lambda}
$$

To see that the mappings $v_{\lambda}^{\tau}$ do form a mapping of $X$ to $Z$, one has to show that, for $(\lambda, \tau) \leq\left(\lambda^{\prime}, \tau^{\prime}\right)$,

$$
r_{\lambda \lambda^{\prime}}^{\tau \tau^{\prime}} v_{\lambda^{\prime}}^{\tau^{\prime}}=v_{\lambda}^{\tau}
$$

Because of (1.1), the verification of (1.5) reduces to the verification of $r_{\lambda \lambda^{\prime}}^{\tau^{\prime}} v_{\lambda^{\prime}}^{\tau^{\prime}}=v_{\lambda}^{\tau^{\prime}}$ and $r_{\lambda}^{\tau \tau^{\prime}} v_{\lambda}^{\tau^{\prime}}=v_{\lambda}^{\tau}$. However, by (1.4) and (1.2), $r_{\lambda \lambda^{\prime}}^{\tau^{\prime}} v_{\lambda^{\prime}}^{\tau^{\prime}}=$ $r_{\lambda \lambda^{\prime}}^{\tau^{\prime}} u_{\lambda^{\prime}}^{\tau^{\prime}} p_{\lambda^{\prime}}=u_{\lambda}^{\tau^{\prime}} p_{\lambda \lambda^{\prime}} p_{\lambda^{\prime}}=u_{\lambda}^{\tau^{\prime}} p_{\lambda}=v_{\lambda}^{\tau^{\prime}}$. Similarly, by (1.4) and (1.3), $r_{\lambda}^{\tau \tau^{\prime}} v_{\lambda}^{\tau^{\prime}}=r_{\lambda}^{\tau \tau^{\prime}} u_{\lambda}^{\tau^{\prime}} p_{\lambda}=u_{\lambda}^{\tau} p_{\lambda}=v_{\lambda}^{\tau}$.

The purpose of the present paper is to prove the following results.

ThEOREM 1.1. Let $\boldsymbol{X}=\left(X_{\lambda}, p_{\lambda \lambda^{\prime}}, \Lambda\right)$ be a cofinite inverse system of compact Hausdorff spaces. Then there exists a cofinite rectangular inverse system of compact polyhedra $Z=\left(Z_{\lambda}^{\tau}, r_{\lambda \lambda^{\prime}}^{\tau \tau^{\prime}}, \Lambda \times T\right)$ and there exist limits $\boldsymbol{u}_{\lambda}: X_{\lambda} \rightarrow \boldsymbol{Z}_{\lambda}$ of vertical systems of $\boldsymbol{Z}, \lambda \in \Lambda$, such that $\boldsymbol{u}_{\lambda} p_{\lambda \lambda^{\prime}}=\boldsymbol{r}_{\lambda \lambda^{\prime}} \boldsymbol{u}_{\lambda^{\prime}}$, for $\lambda \leq \lambda^{\prime}$.

REMARK 1.2. For a cofinite directed set $\Lambda$, denote by $\mathcal{C}_{\Lambda}$ the category, whose objects are inverse systems of compact Hausdorff spaces indexed by $\Lambda$ and whose morphisms are level mappings between such systems. Then the horizontal systems $\boldsymbol{Z}^{\tau}, \tau \in T$, of a cofinite rectangular system $\boldsymbol{Z}$ of compact Hausdorff spaces and the level mappings $\boldsymbol{r}^{\tau \tau^{\prime}}: \boldsymbol{Z}^{\tau^{\prime}} \rightarrow \boldsymbol{Z}^{\tau}, \tau \leq \tau^{\prime}$, between 
horizontal systems of $\boldsymbol{Z}$ form an inverse system $\boldsymbol{Z}^{*}$ in $\mathcal{C}_{\Lambda}$. By Theorem 1.1, the mappings $\boldsymbol{u}^{\tau}: \boldsymbol{X} \rightarrow \boldsymbol{Z}^{\tau}, \tau \in T$, form a limit $\boldsymbol{u}^{*}: \boldsymbol{X} \rightarrow \boldsymbol{Z}^{*}$ of $\boldsymbol{Z}^{*}$ in $\mathcal{C}_{\Lambda}$.

Corollary 1.3. Let $\boldsymbol{X}=\left(X_{n}, p_{n n^{\prime}}, \mathbb{N}\right)$ be an inverse sequence of metric compacta. Then there exists a rectangular inverse system of compact polyhedra $\boldsymbol{Z}=\left(Z_{n}^{m}, r_{n n^{\prime}}^{m m^{\prime}}, \mathbb{N} \times \mathbb{N}\right)$ and there exist limits $\boldsymbol{u}_{n}: X_{n} \rightarrow \boldsymbol{Z}_{n}$ of vertical sequences of $\boldsymbol{Z}, n \in \mathbb{N}$, such that $\boldsymbol{u}_{n} p_{n n^{\prime}}=\boldsymbol{r}_{n n^{\prime}} \boldsymbol{u}_{n^{\prime}}$, for $n \leq n^{\prime}$.

REMARK 1.4. If $\boldsymbol{X}=\left(X_{n}, p_{n n^{\prime}}, \mathbb{N}\right)$ is an inverse sequence of metric compacta, $\boldsymbol{Z}_{n}=\left(Z_{n}^{m}, r_{n}^{m m^{\prime}}, \mathbb{N}\right), n \in \mathbb{N}$, are inverse sequences of compact polyhedra and $\boldsymbol{u}_{n}=\left(u_{n}^{m}\right): X_{n} \rightarrow \boldsymbol{Z}_{n}$ are limits, then it is not always possible to define horizontal mappings $r_{n n^{\prime}}^{m}: Z_{n^{\prime}}^{m} \rightarrow Z_{n}^{m}$ and obtain a rectangular inverse system $\boldsymbol{Z}=\left(Z_{n}^{m}, r_{n n^{\prime}}^{m m^{\prime}}, \mathbb{N} \times \mathbb{N}\right)$ such that $\boldsymbol{u}_{n} p_{n n^{\prime}}=\boldsymbol{r}_{n n^{\prime}} \boldsymbol{u}_{n^{\prime}}$, for $n \leq n^{\prime}$. A simple example is obtained as follows (see [8], pages 363-364). Let $X_{1}=I=[0,1]$, let $X_{2}=C$ be the Cantor set and let $p_{12}: C \rightarrow[0,1]$ be the well-known Cantor mapping. Let $\boldsymbol{Z}_{1}$ consists of copies of $I$ with identities as bonding mappings and let $\boldsymbol{u}_{1}: X_{1} \rightarrow \boldsymbol{Z}_{1}$ be given also by identity mappings. Let $\boldsymbol{u}_{2}: X_{2} \rightarrow \boldsymbol{Z}_{2}$ be the standard expansion of $C$ into an inverse sequence of finite sets. Then there is no level mapping $\boldsymbol{r}_{12}: \boldsymbol{Z}_{2} \rightarrow \boldsymbol{Z}_{1}$ such that $\boldsymbol{u}_{1} p_{12}=\boldsymbol{r}_{12} \boldsymbol{u}_{2}$. Indeed, $p_{12}$ is surjective and thus, $p_{12}=r_{12}^{m} u_{2}^{m}$ would imply that also $r_{12}^{m}: Z_{2}^{m} \rightarrow I$ is surjective, which is not the case, because $r_{12}^{m}\left(Z_{2}^{m}\right)$ is a finite set.

TheOREm 1.5. Let $\boldsymbol{X}, \boldsymbol{Z}$ and $\boldsymbol{u}_{\lambda}, \lambda \in \Lambda$, have all the properties stated in Theorem 1.1. If $\boldsymbol{p}=\left(p_{\lambda}\right): X \rightarrow \boldsymbol{X}$ is a limit of $\boldsymbol{X}$, then the induced mapping $\boldsymbol{v}: X \rightarrow \boldsymbol{Z}$ is a limit of $\boldsymbol{Z}$.

The following corollary is an immediate consequence of Theorems 1.1 and 1.5 .

COROllary 1.6. Let $\boldsymbol{X}=\left(X_{\lambda}, p_{\lambda \lambda^{\prime}}, \Lambda\right)$ be a cofinite inverse system of compact Hausdorff spaces with limit space $X$. Then there exists a cofinite rectangular inverse system of compact polyhedra $\boldsymbol{Z}=\left(Z_{\lambda}^{\tau}, r_{\lambda \lambda^{\prime}}^{\tau \tau^{\prime}}, \Lambda \times T\right)$ such that, for every $\lambda \in \Lambda$, the limit space of the vertical system $\boldsymbol{Z}_{\lambda}$ is $X_{\lambda}$ and the limit space of $\boldsymbol{Z}$ is $X$.

ThEOREM 1.7. Let $\boldsymbol{X}=\left(X_{\lambda}, p_{\lambda \lambda^{\prime}}, \Lambda\right)$ be a cofinite inverse system of topological spaces. Then there exists a cofinite rectangular inverse system of ANRs for metric spaces (of polyhedra) $\boldsymbol{Z}=\left(Z_{\lambda}^{\tau}, r_{\lambda \lambda^{\prime}}^{\tau \tau^{\prime}}, \Lambda \times T\right)$ and there exist resolutions $\boldsymbol{u}_{\lambda}: X_{\lambda} \rightarrow \boldsymbol{Z}_{\lambda}, \lambda \in \Lambda$, such that $\boldsymbol{u}_{\lambda} p_{\lambda \lambda^{\prime}}=\boldsymbol{r}_{\lambda \lambda^{\prime}} \boldsymbol{u}_{\lambda^{\prime}}$, for $\lambda \leq \lambda^{\prime}$.

REMARK 1.8. For a cofinite directed set $\Lambda$, denote by $\mathcal{S}_{\Lambda}$ the category, whose objects are inverse systems of topological spaces indexed by $\Lambda$ and whose morphisms are level mappings between such systems. Then the horizontal systems $\boldsymbol{Z}^{\tau}, \tau \in T$, of a cofinite rectangular system $\boldsymbol{Z}$ of topological spaces and the level mappings $\boldsymbol{r}^{\tau \tau^{\prime}}: \boldsymbol{Z}^{\tau^{\prime}} \rightarrow \boldsymbol{Z}^{\tau}, \tau \leq \tau^{\prime}$, between horizontal systems of $\boldsymbol{Z}$ form an inverse system $Z^{*}$ in $\mathcal{S}_{\Lambda}$. Moreover, the mappings 
$\boldsymbol{u}^{\tau}: \boldsymbol{X} \rightarrow \boldsymbol{Z}^{\tau}, \tau \in T$, form a mapping $\boldsymbol{u}^{*}: \boldsymbol{X} \rightarrow \boldsymbol{Z}^{*}$ in $\mathcal{S}_{\Lambda}$. By Theorem 1.7, $\boldsymbol{u}^{*}$ can be viewed as a resolution of $\boldsymbol{X}$.

Theorem 1.9. Let $\boldsymbol{X}, \boldsymbol{Z}$ and $\boldsymbol{u}_{\lambda}, \lambda \in \Lambda$, have all the properties stated in Theorem 1.7. If $\boldsymbol{p}: X \rightarrow \boldsymbol{X}$ is a resolution of $X$, then the induced mapping $\boldsymbol{v}: X \rightarrow \boldsymbol{Z}$ is also a resolution of $X$.

Corollary 1.6 was conjectured at the 1998 Dubrovnik topology conference by Yu.T. Lisitsa (see [1]). The result was also quoted in Lisitsa's recent paper on strong shape of compact Hausdorff spaces ([2], Theorem 1). For the proof he refers to [6]. However, in [6] (and also in [7]) only a weaker assertion was proved, because the systems $\boldsymbol{Z}$ constructed in those papers were not rectangular. More precisely, the index set of $\boldsymbol{Z}$ was the disjoint union $\bigsqcup_{\lambda \in \Lambda} T_{\lambda}$ of a family of (possibly different) cofinite sets $T_{\lambda}$. A weaker version of Corollary 1.6 appears in [3] as Theorem 4. In that version strict commutativity of some of the diagrams is replaced by commutativity up to homotopy. Theorems 1.7 and 1.9 generalize Theorems 1.1 and 1.5 to arbitrary topological spaces. It is well known that in non-compact situations inverse limits must be replaced by resolutions (see [5] and [4]).

The author wishes to thank the referee for suggesting that methods developed in this paper and a result of N. Uglešić [7] could be used to obtain the polyhedral version of Theorem 1.7.

\section{SOME FACTS ON LIMITS AND RESOLUTIONS}

In this section we recall some facts concerning inverse systems of topological spaces. We consider systems $\boldsymbol{X}=\left(X_{\lambda}, p_{\lambda \lambda^{\prime}}, \Lambda\right)$, indexed by directed ordered sets $\Lambda$. If the index set $\Lambda$ is cofinite, i.e., every element has only finitely many predecessors, we speak of a cofinite inverse system. A mapping $\boldsymbol{f}=\left(f, f_{\mu}\right): \boldsymbol{X} \rightarrow \boldsymbol{Y}$ between inverse systems $\boldsymbol{X}$ and $\boldsymbol{Y}=\left(Y_{\mu}, q_{\mu \mu^{\prime}}, M\right)$ consists of a function $f: M \rightarrow \Lambda$ and of mappings $f_{\mu}: X_{f(\mu)} \rightarrow Y_{\mu}, \mu \in M$, such that, for $\mu \leq \mu^{\prime}$, there exists a $\lambda \geq f(\mu), f\left(\mu^{\prime}\right)$ such that

$$
f_{\mu} p_{f(\mu) \lambda}=q_{\mu \mu^{\prime}} f_{\mu^{\prime}} p_{f\left(\mu^{\prime}\right) \lambda} .
$$

A mapping $\boldsymbol{f}=\left(f, f_{\mu}\right)$ is called special if $f$ is increasing and

$$
f_{\mu} p_{f(\mu) f\left(\mu^{\prime}\right)}=q_{\mu \mu^{\prime}} f_{\mu^{\prime}} .
$$

A special mappig, where $f$ is the identity function, is called a level mapping and is denoted by $\boldsymbol{f}=\left(f_{\lambda}\right)$. Composition $\boldsymbol{h}=\boldsymbol{g} \boldsymbol{f}$ of mappings $\boldsymbol{f}$ and $\boldsymbol{g}=$ $\left(g, g_{\mu}\right): \boldsymbol{Y} \rightarrow \boldsymbol{Z}=\left(Z_{\nu}, r_{\nu \nu^{\prime}}, N\right)$ consists of the function $h=f g: M \rightarrow \Lambda$ and of the mappings $h_{\nu}=g_{\nu} f_{g(\nu)}: X_{h(\nu)} \rightarrow Z_{\nu}$. The identity mapping $\mathbf{1}_{\boldsymbol{Y}}: \boldsymbol{Y} \rightarrow$ $\boldsymbol{Y}$ consists of the identity function $1_{M}: M \rightarrow M$ and of the identity mappings $1_{\mu}: Y_{\mu} \rightarrow Y_{\mu}$.

A mapping $\boldsymbol{f}=\left(f_{\mu}\right): X \rightarrow \boldsymbol{Y}$ of a space $X$ to a system $\boldsymbol{Y}$ consists of mappings $f_{\mu}: X \rightarrow Y_{\mu}, \mu \in M$, such that $q_{\mu \mu^{\prime}} f_{\mu^{\prime}}=f_{\mu}$, for $\mu \leq \mu^{\prime}$. A limit of 
a system $\boldsymbol{Y}$ is a mapping $\boldsymbol{q}=\left(q_{\mu}\right): Y \rightarrow \boldsymbol{Y}$, which has the following universal property: for any mapping $\boldsymbol{f}: X \rightarrow \boldsymbol{Y}$, there exists a unique mapping $f: X \rightarrow$ $Y$ such that $\boldsymbol{q} f=\boldsymbol{f}$. Limits always exist and are unique up to natural isomorphism. If in a system $\boldsymbol{Y}$ all terms $Y_{\mu}$ are compact Hausdorff spaces, then so is the limit space $Y$. Every compact Hausdorff space is the limit space of an inverse system of compact polyhedra.

Resolutions of a space $X$ are mappings $\boldsymbol{p}=\left(p_{\lambda}\right): X \rightarrow \boldsymbol{X}=\left(X_{\lambda}, p_{\lambda \lambda^{\prime}}, \Lambda\right)$, which satisfy two additional conditions (see [5], I,6.1, or [4], II,6):

(B1) For every normal (numerable) covering $\mathcal{U}$ of $X$, there is a $\lambda \in \Lambda$ and there is a normal covering $\mathcal{U}_{\lambda}$ of $X_{\lambda}$ such that the covering $p_{\lambda}^{-1}\left(\mathcal{U}_{\lambda}\right)$ refines $\mathcal{U}$.

(B2) For every $\lambda \in \Lambda$ and every normal covering $\mathcal{U}_{\lambda}$ of $X_{\lambda}$, there is a $\lambda^{\prime} \geq \lambda$ such that

$$
p_{\lambda \lambda^{\prime}}\left(X_{\lambda^{\prime}}\right) \subseteq \operatorname{St}\left(p_{\lambda}(X), \mathcal{U}_{\lambda}\right) .
$$

If all $X_{\lambda}$ are normal spaces, condition (B2) can be replaced by the equivalent condition:

(B2) For every $\lambda \in \Lambda$ and every open neighborhood $U$ of the closure $\overline{p_{\lambda}(X)}$ in $X_{\lambda}$, there is a $\lambda^{\prime} \geq \lambda$ such that

$$
p_{\lambda \lambda^{\prime}}\left(X_{\lambda^{\prime}}\right) \subseteq U
$$

It is well known that, for $X_{\lambda}$ Tychonoff and $X$ topologically complete (e.g., for $X$ paracompact), every resolution $\boldsymbol{p}: X \rightarrow \boldsymbol{X}$ is an inverse limit. Furthermore, if $X$ and $X_{\lambda}$ are compact Hausdorff spaces, also the converse holds, i.e., if $\boldsymbol{p}: X \rightarrow \boldsymbol{X}$ is an inverse limit, then $\boldsymbol{p}$ is a resolution. It is also known that every topological space $X$ admits an ANR-resolution (a polyhedral resolution) $\boldsymbol{p}: X \rightarrow \boldsymbol{X}$, i.e., a resolution such that all $X_{\lambda}$ are ANR's for metric spaces (are polyhedra with the CW-topology). For the proofs of these results see, e.g., [5] or [4].

The following cofinality lemma will prove useful in the next section.

Lemma 2.1. Let $\boldsymbol{X}=\left(X_{\lambda}, p_{\lambda \lambda^{\prime}}, \Lambda\right)$ and $\boldsymbol{Y}=\left(Y_{\mu}, q_{\mu \mu^{\prime}}, M\right)$ be inverse systems of topological spaces and let $\boldsymbol{f}=\left(f, f_{\mu}\right): \boldsymbol{X} \rightarrow \boldsymbol{Y}$ be a special mapping of systems such that the function $f: M \rightarrow \Lambda$ is cofinal and every $f_{\mu}: X_{f(\mu)} \rightarrow$ $Y_{\mu}, \mu \in M$, is a homeomorphism. If $\boldsymbol{p}: X \rightarrow \boldsymbol{X}$ is a resolution, then the composition $\boldsymbol{q}=\boldsymbol{f} \boldsymbol{p}: X \rightarrow \boldsymbol{Y}$ is also a resolution.

Proof. If $\boldsymbol{p}$ consists of mappings $p_{\lambda}: X \rightarrow X_{\lambda}, \lambda \in \Lambda, \boldsymbol{q}$ consists of mappings $q_{\mu}=f_{\mu} p_{f(\mu)}: X \rightarrow Y_{\mu}, \mu \in M$. Let $\mathcal{U}$ be a normal covering of $X$. Since $\boldsymbol{p}$ is a resolution, there exist a $\lambda \in \Lambda$ and a normal covering $\mathcal{U}_{\lambda}$ of $X_{\lambda}$ such that $\left(p_{\lambda}\right)^{-1}\left(\mathcal{U}_{\lambda}\right)$ refines $\mathcal{U}$. Since $f$ is cofinal, there exists a $\mu \in M$ such that $\lambda \leq f(\mu)$. Since $f_{\mu}: X_{f(\mu)} \rightarrow Y_{\mu}$ is a homeomorphism, there exists a normal covering $\mathcal{V}_{\mu}$ of $Y_{\mu}$ such that $f_{\mu}^{-1}\left(\mathcal{V}_{\mu}\right)=p_{\lambda f(\mu)}^{-1}\left(\mathcal{U}_{\lambda}\right)$. Consequently, 
$q_{\mu}^{-1}\left(\mathcal{V}_{\mu}\right)=p_{f(\mu)}^{-1} f_{\mu}^{-1}\left(\mathcal{V}_{\mu}\right)=p_{f(\mu)}^{-1} p_{\lambda f(\mu)}^{-1}\left(\mathcal{U}_{\lambda}\right)=p_{\lambda}^{-1}\left(\mathcal{U}_{\lambda}\right)$ refines $\mathcal{U}$, which is property (B1) for $\boldsymbol{q}$.

To verify property (B2), consider $\mu \in M$ and a normal covering $\mathcal{V}_{\mu}$ of $Y_{\mu}$. Then consider the normal covering $f_{\mu}^{-1}\left(\mathcal{V}_{\mu}\right)$ of $X_{f(\mu)}$. By property (B2) for $\boldsymbol{p}$, there exists an index $\lambda \geq f(\mu)$ such that $p_{f(\mu) \lambda}\left(X_{\lambda}\right) \subseteq \operatorname{St}\left(p_{f(\mu)}(X), f_{\mu}^{-1}\left(\mathcal{V}_{\mu}\right)\right)$. Since $f$ is inceasing and cofinal, there exists a $\mu^{\prime} \geq \mu$ such that $f\left(\mu^{\prime}\right) \geq \lambda$. Moreover, $q_{\mu \mu^{\prime}} f_{\mu^{\prime}}=f_{\mu} p_{f(\mu) f\left(\mu^{\prime}\right)}$, because $\boldsymbol{f}$ is a special mapping. Since $f_{\mu^{\prime}}$ is a homeomorphism, $Y_{\mu^{\prime}}=f_{\mu^{\prime}}\left(X_{f\left(\mu^{\prime}\right)}\right)$. Therefore, taking into account the fact that $q_{\mu}=f_{\mu} p_{f(\mu)}$, one concludes that

$$
\begin{aligned}
& q_{\mu \mu^{\prime}}\left(Y_{\mu^{\prime}}\right)=q_{\mu \mu^{\prime}} f_{\mu^{\prime}}\left(X_{f\left(\mu^{\prime}\right)}\right)=f_{\mu} p_{f(\mu) f\left(\mu^{\prime}\right)}\left(X_{f\left(\mu^{\prime}\right)}\right) \\
& =f_{\mu} p_{f(\mu) \lambda} p_{\lambda f\left(\mu^{\prime}\right)}\left(X_{f\left(\mu^{\prime}\right)}\right) \subseteq f_{\mu} p_{f(\mu) \lambda}\left(X_{\lambda}\right) \\
& \subseteq f_{\mu}\left(\operatorname{St}\left(p_{f(\mu)}(X), f_{\mu}^{-1}\left(\mathcal{V}_{\mu}\right)\right)\right)=\operatorname{St}\left(q_{\mu}(X), \mathcal{V}_{\mu}\right)
\end{aligned}
$$

which establishes property (B2) for $\boldsymbol{q}$.

\section{Proof of Theorem 1.7 in the ANR Case}

The proof consists of the proof of Theorem 2 in [6], enriched by additional arguments. We give here the entire proof divided in eight steps, referring only for some details in some of the steps to the corresponding parts of [6].

Step 1. Consider the family $\left(X_{\lambda}, \lambda \in \Lambda\right)$ of all terms of $\boldsymbol{X}$. For every $\lambda \in \Lambda$, choose an ANR-resolution $\boldsymbol{e}_{\lambda}=\left(e_{\lambda}^{\delta}\right): X_{\lambda} \rightarrow \boldsymbol{Y}_{\lambda}=\left(Y_{\lambda}^{\delta}, e_{\lambda}^{\delta \delta^{\prime}}, \Delta_{\lambda}\right)$. Let $M=\prod_{\lambda \in \Lambda} \Delta_{\lambda}$ be the product of the ordered sets $\Delta_{\lambda}$, i.e., elements of $M$ are functions $\mu$ with domain $\Lambda$ and values $\mu(\lambda) \in \Delta_{\lambda}$, for all $\lambda \in \Lambda$. Put $\mu \leq \mu^{\prime}$ whenever $\mu(\lambda) \leq \mu^{\prime}(\lambda)$, for all $\lambda \in \Lambda$. Note that $M$ is a directed ordered set. Now put $X_{\lambda}^{\mu}=Y_{\lambda}^{\mu(\lambda)}, p_{\lambda}^{\mu \mu^{\prime}}=e_{\lambda}^{\mu(\lambda) \mu^{\prime}(\lambda)}$ and $p_{\lambda}^{\mu}=e_{\lambda}^{\mu(\lambda)}$. Then $\boldsymbol{X}_{\lambda}=\left(X_{\lambda}^{\mu}, p_{\lambda}^{\mu \mu^{\prime}}, M\right)$ is an inverse system and the mappings $p_{\lambda}^{\mu}: X_{\lambda} \rightarrow X_{\lambda}^{\mu}$ form a mapping $\boldsymbol{p}_{\lambda}: X_{\lambda} \rightarrow \boldsymbol{X}_{\lambda}$. Let $\boldsymbol{f}: \boldsymbol{Y}_{\lambda} \rightarrow \boldsymbol{X}_{\lambda}$ be the special mapping give by the increasing function $f: M \rightarrow \Delta_{\lambda}$, where $f(\mu)=\mu(\lambda)$, and by the identity mappings $f^{\mu}: Y_{\lambda}^{f(\mu)}=Y_{\lambda}^{\mu(\lambda)} \rightarrow Y_{\lambda}^{\mu(\lambda)}=X_{\lambda}^{\mu}$. Note that $f$ is cofinal, because, for any $\delta \in \Delta_{\lambda}$, any function $\mu$ such that $\mu(\lambda)=\delta$ and $\mu\left(\lambda^{\prime}\right) \in$ $\Delta_{\lambda^{\prime}}$, for $\lambda^{\prime} \in \Lambda$, belongs to $M$ and has the property that $f(\mu)=\delta$. Since $\boldsymbol{e}_{\lambda}: X_{\lambda} \rightarrow \boldsymbol{Y}_{\lambda}$ is a resolution, Lemma 2.1 implies that also $\boldsymbol{f} \boldsymbol{e}_{\lambda}: Y_{\lambda} \rightarrow \boldsymbol{X}_{\lambda}$ is

a resolution. However, $\boldsymbol{f} \boldsymbol{e}_{\lambda}$ consists of mappings $f^{\mu} e_{\lambda}^{f(\mu)}=e_{\lambda}^{\mu(\lambda)}=p_{\lambda}^{\mu}$, which shows that $\boldsymbol{f} \boldsymbol{e}_{\lambda}=\boldsymbol{p}_{\lambda}$ and thus, $\boldsymbol{p}_{\lambda}$ is a resolution. Note that all systems $\boldsymbol{X}_{\lambda}$, $\lambda \in \Lambda$, are indexed by the same set $M$.

STEP 2. For $(\lambda, \mu) \in \Lambda \times M$, consider the product $Y_{\lambda}^{\mu}=\prod_{\zeta \leq \lambda} X_{\zeta}^{\mu}$. Since $\Lambda$ is cofinite, every set $\{\zeta \in \Lambda \mid \zeta \leq \lambda\}$ is finite and therefore, $Y_{\lambda}^{\mu}$ is an ANR. Also consider the mappings $q_{\lambda}^{\mu \mu^{\prime}}=\prod_{\zeta \leq \lambda} p_{\zeta}^{\mu \mu^{\prime}}: Y_{\lambda}^{\mu^{\prime}} \rightarrow Y_{\lambda}^{\mu}$, defined for $\mu \leq \mu^{\prime}$. Then $\boldsymbol{Y}_{\lambda}=\left(Y_{\lambda}^{\mu}, q_{\lambda}^{\mu \mu^{\prime}}, M\right)$ is an inverse system. Moreover, the mappings $p_{\zeta}^{\mu} p_{\zeta \lambda}: X_{\lambda} \rightarrow X_{\lambda}^{\mu}, \zeta \leq \lambda$, into factors of $Y_{\lambda}^{\mu}$, determine mappings $q_{\lambda}^{\mu}: X_{\lambda} \rightarrow Y_{\lambda}^{\mu}$, which form a mapping $\boldsymbol{q}_{\lambda}: X_{\lambda} \rightarrow \boldsymbol{Y}_{\lambda}$. We claim that $\boldsymbol{q}_{\lambda}$ 
has property (B1) (see Lemma 5 in [6]). Indeed, If $\mathcal{U}$ is a normal covering of $X_{\lambda}$, by property (B1) for $\boldsymbol{p}_{\lambda}$, there exists an index $\mu \in M$ and an open covering $\mathcal{V}$ of $X_{\lambda}^{\mu}$ such that $\left(p_{\lambda}^{\mu}\right)^{-1}(\mathcal{V})$ refines $\mathcal{U}$. Consider the open covering $\mathcal{W}$ of $Y_{\lambda}^{\mu}$, consisting of the sets $W=\left(\prod_{\zeta<\lambda} X_{\zeta}^{\mu}\right) \times V$, where $V \in \mathcal{V}$. Clearly, $\left(q_{\lambda}^{\mu}\right)^{-1}(\mathcal{W})=\left(p_{\lambda}^{\mu}\right)^{-1}(\mathcal{V})$ and thus, it refines $\mathcal{U}$.

STEP 3. Order $\Lambda \times M$ by putting $\nu=(\lambda, \mu) \leq\left(\lambda^{\prime}, \mu^{\prime}\right)=\nu^{\prime}$, whenever $\lambda \leq \lambda^{\prime}$ and $\mu \leq \mu^{\prime}$. Define a rectangular inverse system of ANR's $\boldsymbol{Y}=$ $\left(Y_{\nu}, q_{\nu \nu^{\prime}}, \Lambda \times M\right)$ as follows. Put $Y_{\nu}=Y_{\lambda}^{\mu}$ and for $\nu \leq \nu^{\prime}$, let $q_{\nu \nu^{\prime}}: Y_{\lambda^{\prime}}^{\mu^{\prime}} \rightarrow Y_{\lambda}^{\mu}$ be the composition of two mappings. The first one is the natural projection $Y_{\lambda^{\prime}}^{\mu^{\prime}}=\prod_{\zeta \leq \lambda^{\prime}} X_{\zeta}^{\mu^{\prime}} \rightarrow \prod_{\zeta \leq \lambda} X_{\zeta}^{\mu^{\prime}}=Y_{\lambda}^{\mu^{\prime}}$ and the second one is the mapping $q_{\lambda}^{\mu \mu^{\prime}}: Y_{\lambda}^{\mu^{\prime}} \rightarrow Y_{\lambda}^{\mu}$. Note that

$$
q_{\nu \nu^{\prime}} q_{\lambda^{\prime}}^{\mu^{\prime}}=q_{\lambda}^{\mu} p_{\lambda \lambda^{\prime}}
$$

This is so because, for $\zeta \leq \lambda$, the $\zeta$-coordinate mapping of the left side of (3.1) equals $p_{\zeta}^{\mu \mu^{\prime}} p_{\zeta}^{\mu^{\prime}} p_{\zeta \lambda^{\prime}}=p_{\zeta}^{\mu} p_{\zeta \lambda^{\prime}}$, while the $\zeta$-coordinate mapping of the right side of (3.1) equals $p_{\zeta}^{\mu} p_{\zeta \lambda} p_{\lambda \lambda^{\prime}}=p_{\zeta}^{\mu} p_{\zeta \lambda^{\prime}}$. Also note that, for $\lambda=\lambda^{\prime}, q_{\nu \nu^{\prime}}=q_{\lambda}^{\mu \mu^{\prime}}$.

STEP 4. For $\lambda \in \Lambda$, let $\Gamma_{\lambda}$ be the set of all pairs $\gamma=(\mu, G)$, where $\mu \in M$ and $G$ is an open neighborhood of the closure $\overline{q_{\lambda}^{\mu}\left(X_{\lambda}\right)}$ in $Y_{\lambda}^{\mu}$. Since $Y_{\lambda}^{\mu}$ is an ANR, so is $G$. Order $\Gamma_{\lambda}$ by putting $\gamma \leq \gamma^{\prime}=\left(\mu^{\prime}, G^{\prime}\right)$, whenever $\mu \leq \mu^{\prime}$ and $q_{\lambda}^{\mu \mu^{\prime}}\left(G^{\prime}\right) \subseteq G$. Put $S_{\lambda}^{\gamma}=G$ and define $s_{\lambda}^{\gamma \gamma^{\prime}}: S_{\lambda}^{\gamma^{\prime}} \rightarrow S_{\lambda}^{\gamma}, \gamma \leq \gamma^{\prime}$, as the restriction $q_{\lambda}^{\mu \mu^{\prime}} \mid G^{\prime}: G^{\prime} \rightarrow G$ of $q_{\lambda}^{\mu \mu^{\prime}}$. Then $\boldsymbol{S}_{\lambda}=\left(S_{\lambda}^{\gamma}, s_{\lambda}^{\gamma \gamma^{\prime}}, \Gamma_{\lambda}\right)$ is an inverse system of ANRs. The restrictions of the mappings $q_{\lambda}^{\mu}: X_{\lambda} \rightarrow Y_{\lambda}^{\mu}$ to the codomains $G$ are mappings $s_{\lambda}^{\gamma}: X_{\lambda} \rightarrow S_{\lambda}^{\gamma}$, which form a mapping $\boldsymbol{s}_{\lambda}: X_{\lambda} \rightarrow \boldsymbol{S}_{\lambda}$. Moreover, $\boldsymbol{s}_{\lambda}$ is a resolution (see Lemma 2 in [6] or Lemma 6.24 of [4]). Indeed, if $\mathcal{U}$ is a normal covering of $X_{\lambda}$, then (B1) for $\boldsymbol{q}_{\lambda}$ yields a $\mu \in M$ and a normal covering $\mathcal{V}$ of $Y_{\lambda}^{\mu}$ such that $\left(q_{\lambda}^{\mu}\right)^{-1}(\mathcal{V})$ refines $\mathcal{U}$. However, the pair $\gamma=\left(\mu, Y_{\lambda}^{\mu}\right)$ belongs to $\Gamma_{\lambda}, S_{\lambda}^{\gamma}=Y_{\lambda}^{\mu}$ and $s_{\lambda}^{\gamma}=q_{\lambda}^{\mu}$. Therefore, $\mathcal{V}$ is an open covering of $S_{\lambda}^{\gamma}$ and $\left(s_{\lambda}^{\gamma}\right)^{-1}(\mathcal{V})$ refines $\mathcal{U}$, which proves (B1) for $\boldsymbol{s}_{\lambda}$. Now assume that $\gamma=(\mu, G) \in \Gamma_{\lambda}$ and $U$ is an open neighborhood of the closure of $s_{\lambda}^{\gamma}\left(X_{\lambda}\right)$ in $S_{\lambda}^{\gamma}=G$. Clearly, this closure coincides with the closure of $q_{\lambda}^{\mu}\left(X_{\lambda}\right)$ in $Y_{\lambda}^{\mu}$. Therefore, $\gamma^{\prime}=(\mu, U)$ also belongs to $\Gamma_{\lambda}$ and $\gamma \leq \gamma^{\prime}$, because $q_{\lambda}^{\mu \mu}(U)=U \subseteq G$. However, $Y_{\lambda}^{\gamma^{\prime}}=U$ and $s_{\lambda}^{\gamma \gamma^{\prime}}\left(Y_{\lambda}^{\gamma^{\prime}}\right)=q_{\lambda}^{\mu \mu}(U)=U$, which shows that $s_{\lambda}$ also has property $(\mathrm{B} 2)^{\prime}$.

STEP 5. Consider the disjoint union $\Gamma=\coprod_{\lambda \in \Lambda} \Gamma_{\lambda}$ and order it by putting $\gamma=(\mu, G) \leq\left(\mu^{\prime}, G^{\prime}\right)=\gamma^{\prime}$, for $\gamma \in \Gamma_{\lambda}, \gamma^{\prime} \in \Gamma_{\lambda^{\prime}}$, whenever $\nu=(\lambda, \mu) \leq$ $\left(\lambda^{\prime}, \mu^{\prime}\right)=\nu^{\prime}$ in $\Lambda \times M$ and $q_{\nu \nu^{\prime}}\left(G^{\prime}\right) \subseteq G$. Note that the restriction of this ordering to $\Gamma_{\lambda}$ coincides with the previously defined ordering on $\Gamma_{\lambda}$. Also note that for every $\gamma \in \Gamma_{\lambda}$ and every $\lambda^{\prime} \geq \lambda$, there exists a $\gamma^{\prime} \in \Gamma_{\lambda^{\prime}}$ such that $\gamma \leq \gamma^{\prime}$ in $\Gamma$. Indeed, if $\gamma=(\mu, G)$, then $G$ is an open neighborhood of $\overline{q_{\lambda}^{\mu}\left(X_{\lambda}\right)}$ in $Y_{\lambda}^{\mu}$ and $\nu^{\prime}=\left(\lambda^{\prime}, \mu\right) \geq \nu=(\lambda, \mu)$. Since, by $(3.1), q_{\lambda}^{\mu} p_{\lambda \lambda^{\prime}}=q_{\nu \nu^{\prime}} q_{\lambda^{\prime}}^{\mu}$, 
we see that $q_{\nu \nu^{\prime}}\left(\overline{q_{\lambda^{\prime}}^{\mu}\left(X_{\lambda^{\prime}}\right)}\right) \subseteq \overline{q_{\nu \nu^{\prime}}\left(q_{\lambda^{\prime}}^{\mu}\left(X_{\lambda^{\prime}}\right)\right)}=\overline{q_{\lambda}^{\mu}\left(p_{\lambda \lambda^{\prime}}\left(X_{\lambda^{\prime}}\right)\right)} \subseteq \overline{q_{\lambda}^{\mu}\left(X_{\lambda}\right)} \subseteq G$. Consequently, there exists an open neighborhood $G^{\prime}$ of $\overline{q_{\lambda^{\prime}}^{\mu}\left(X_{\lambda^{\prime}}\right)}$ in $Y_{\lambda^{\prime}}^{\mu^{\prime}}$ such that $q_{\nu \nu^{\prime}}\left(G^{\prime}\right) \subseteq G$. This means that $\gamma^{\prime}=\left(\mu, G^{\prime}\right) \in \Gamma_{\lambda^{\prime}}$ has the property that $\gamma \leq \gamma^{\prime}$ in $\Gamma$. It is now easy to see that $\Gamma$ is directed. Indeed, if $\gamma_{i}=$ $\left(\mu_{i}, G_{i}\right) \in \Gamma_{\lambda_{i}}$, for $i=1,2$, and we choose $\lambda^{\prime} \geq \lambda_{1}, \lambda_{2}$, then there exist elements $\gamma_{1}^{\prime}, \gamma_{2}^{\prime} \in \Gamma_{\lambda^{\prime}}$ such that $\gamma_{i} \leq \gamma_{i}^{\prime}, i=1,2$. Since $\Gamma_{\lambda^{\prime}}$ is directed, there exists an element $\gamma^{\prime} \in \Gamma_{\lambda^{\prime}}$ such that $\gamma_{i}^{\prime} \leq \gamma^{\prime}, i=1,2$, and thus, $\gamma_{i} \leq \gamma^{\prime}$, $i=1,2$.

We now define an inverse system $\boldsymbol{S}=\left(S_{\gamma}, s_{\gamma \gamma^{\prime}}, \Gamma\right)$, consisting of ANRs, as follows. If $\gamma=(\mu, G) \in \Gamma_{\lambda}$, we put $S_{\gamma}=S_{\lambda}^{\gamma}=G$ and if $\gamma^{\prime}=\left(\mu^{\prime}, G^{\prime}\right) \in \Gamma_{\lambda^{\prime}}$, $\gamma \leq \gamma^{\prime}$, we define $s_{\gamma \gamma^{\prime}}: S_{\gamma^{\prime}} \rightarrow S_{\gamma}$ as the restriction $q_{\nu \nu^{\prime}} \mid G^{\prime}: G^{\prime} \rightarrow G$ of $q_{\nu \nu^{\prime}}: Y_{\lambda^{\prime}}^{\mu^{\prime}} \rightarrow Y_{\lambda}^{\mu}$. Note that, for $\lambda=\lambda^{\prime}, s_{\gamma \gamma^{\prime}}=s_{\lambda}^{\gamma \gamma^{\prime}}$, because $s_{\gamma \gamma^{\prime}}$ is the restriction of $q_{\nu \nu^{\prime}}$ to the domain $G^{\prime}$ and the codomain $G$, while $s_{\lambda}^{\gamma \gamma^{\prime}}$ is the corresponding restriction of $q_{\lambda}^{\mu \mu^{\prime}}$. However, for $\lambda=\lambda^{\prime}, q_{\nu \nu^{\prime}}=q_{\lambda}^{\mu \mu^{\prime}}$. Also note that (3.1) implies

$$
s_{\gamma \gamma^{\prime}} s_{\lambda^{\prime}}^{\gamma^{\prime}}=s_{\lambda}^{\gamma} p_{\lambda \lambda^{\prime}}
$$

In the special case when $\lambda=\lambda^{\prime},(3.2)$ becomes $s_{\gamma \gamma^{\prime}} s_{\lambda}^{\gamma^{\prime}}=s_{\lambda}^{\gamma}$.

STEP 6. Let $\tilde{T}$ be the set of all functions $\tau: \Lambda \rightarrow \Gamma$ such that $\tau(\lambda) \in \Gamma_{\lambda}$, for $\lambda \in \Lambda$. Order $\tilde{T}$ by putting $\tau \leq \tau^{\prime}$, whenever $\tau(\lambda) \leq \tau^{\prime}(\lambda)$, for all $\lambda \in \Lambda$. The subset $T \subseteq \tilde{T}$, consisting of all increasing functions $\tau$, is cofinal in $\tilde{T}$, i.e., for an arbitrary $\tau_{0} \in \tilde{T}$, there exists an increasing function $\tau: \Lambda \rightarrow \Gamma$ such that $\tau(\lambda) \in \Gamma_{\lambda}$ and $\tau_{0}(\lambda) \leq \tau(\lambda)$, for all $\lambda \in \Lambda$. Indeed, we can construct such a function $\tau$, defining $\tau(\lambda)$, by induction on the number of predecessors of $\lambda$. This number is finite, because $\Lambda$ is cofinite. If the number of predecessors of $\lambda$ is 1 , we put $\tau(\lambda)=\tau_{0}(\lambda)$. Now assume that $\lambda_{1}, \ldots, \lambda_{n}$ are all predecessors of $\lambda$ and that we have already defined $\tau\left(\lambda_{i}\right) \in \Gamma_{\lambda_{i}}$ so that $\tau_{0}\left(\lambda_{i}\right) \leq \tau\left(\lambda_{i}\right)$, for $i \in\{1, \ldots, n\}$. Since $\lambda_{i} \leq \lambda$, by Step 5, there are elements $\gamma_{i} \in \Gamma_{\lambda}$ such that $\tau\left(\lambda_{i}\right) \leq \gamma_{i}$ in $\Gamma$. Now put $\tau(\lambda)=\gamma$, where $\gamma \in \Gamma_{\lambda}$ and $\gamma \geq \gamma_{i}$, for $i \in\{1, \ldots, n\}$, and $\gamma \geq \tau_{0}(\lambda)$. Such a $\gamma$ exists because $\Gamma_{\lambda}$ is directed. It is now easy to conclude that the set $T$ is directed. Indeed, $\tilde{T}$ is directed, because the sets $\Gamma_{\lambda}$ are directed. Therefore, if $\tau_{1}, \tau_{2} \in T \subseteq \tilde{T}$, there exists a function $\tau_{0} \in \tilde{T}$ such that $\tau_{1}, \tau_{2} \leq \tau_{0}$. By cofinality of $T$ in $\tilde{T}$, there is a function $\tau \in T$ such that $\tau_{0} \leq \tau$ and thus, $\tau_{1}, \tau_{2} \leq \tau$.

We now define an inverse system $\boldsymbol{Z}=\left(Z_{\lambda}^{\tau}, r_{\lambda \lambda^{\prime}}^{\tau \tau^{\prime}}, \Lambda \times T\right)$, whose index set $\Lambda \times T$ is the product of directed sets $\Lambda$ and $T$. For $(\lambda, \tau) \in \Lambda \times T$, put $Z_{\lambda}^{\tau}=S_{\tau(\lambda)}$ and for $(\lambda, \tau) \leq\left(\lambda^{\prime}, \tau^{\prime}\right)$, put $r_{\lambda \lambda^{\prime}}^{\tau \tau^{\prime}}=s_{\tau(\lambda) \tau^{\prime}\left(\lambda^{\prime}\right)}$. Note that $\tau(\lambda) \leq \tau^{\prime}(\lambda)$, because $\tau \leq \tau^{\prime}$, and $\tau^{\prime}(\lambda) \leq \tau^{\prime}\left(\lambda^{\prime}\right)$, because $\tau^{\prime}$ is an increasing function. Consequently, $\tau(\lambda) \leq \tau^{\prime}\left(\lambda^{\prime}\right)$ and the mapping $s_{\tau(\lambda) \tau^{\prime}\left(\lambda^{\prime}\right)}$ is well defined. It readily follows that (1.1) is fulfilled and thus, $\boldsymbol{Z}$ is a rectangular inverse system of ANRs. 
STEP 7. For a fixed $\lambda \in \Lambda$, consider the system $\boldsymbol{S}_{\lambda}=\left(S_{\lambda}^{\gamma}, s_{\lambda}^{\gamma \gamma^{\prime}}, \Gamma_{\lambda}\right)$ and the resolution $\boldsymbol{s}_{\lambda}: X_{\lambda} \rightarrow \boldsymbol{S}_{\lambda}$. Also consider the function $f: T \rightarrow \Gamma_{\lambda}$, defined by $f(\tau)=\tau(\lambda)$. This function is increasing and cofinal. Indeed, $\tau \leq \tau^{\prime}$ implies $\tau(\lambda) \leq \tau^{\prime}(\lambda)$ and for an arbitrary element $\gamma \in \Gamma_{\lambda}$, there exists a function $\tau_{0} \in \tilde{T}$ such that $\tau_{0}(\lambda)=\gamma$. Consequently, by Step 5 , there also exists a $\tau \in T$ such that $\tau \geq \tau_{0}$ and thus, $f(\tau)=\tau(\lambda) \geq \tau_{0}(\lambda)=\gamma$. Now consider the special mapping $\boldsymbol{f}=\left(f, f^{\tau}\right): \boldsymbol{S}_{\lambda} \rightarrow \boldsymbol{Z}_{\lambda}$ to the vertical system $\boldsymbol{Z}_{\lambda}=\left(Z_{\lambda}^{\tau}, r_{\lambda}^{\tau \tau^{\prime}}, T\right)$ of $\boldsymbol{Z}$ at level $\lambda$, where $f^{\tau}: S_{\lambda}^{f(\tau)}=S_{\lambda}^{\tau(\lambda)} \rightarrow S_{\lambda}^{\tau(\lambda)}=S_{\tau(\lambda)}=Z_{\lambda}^{\tau}$ is the identity mapping. That $f$ is indeed a special mapping follows from $f^{\tau} s_{\lambda}^{f(\tau) f\left(\tau^{\prime}\right)}=$ $s_{\lambda}^{\tau(\lambda) \tau^{\prime}(\lambda)}=s_{\tau(\lambda) \tau^{\prime}(\lambda)}=r_{\lambda}^{\tau \tau^{\prime}}=r_{\lambda}^{\tau \tau^{\prime}} f^{\tau^{\prime}}$, for $\tau \leq \tau^{\prime}$. The composition $\boldsymbol{u}_{\lambda}=$ $\boldsymbol{f} \boldsymbol{s}_{\lambda}: X_{\lambda} \rightarrow Z_{\lambda}$ consists of mappings $u_{\lambda}^{\tau}=f^{\tau} s_{\lambda}^{f(\tau)}=s_{\lambda}^{\tau(\lambda)}: X_{\lambda} \rightarrow S_{\lambda}^{\tau(\lambda)}=$ $Z_{\lambda}^{\tau}$. An application of Lemma 2.1 shows that $\boldsymbol{u}_{\lambda}: X_{\lambda} \rightarrow \boldsymbol{Z}_{\lambda}$ is a resolution. The mappings $u_{\lambda}^{\tau}$ satisfy condition (1.3), because $\boldsymbol{u}_{\lambda}: X_{\lambda} \rightarrow \boldsymbol{Z}_{\lambda}$ is a mapping. They also satisfy condition (1.2), because it assumes the form $s_{\lambda}^{\tau(\lambda)} p_{\lambda \lambda^{\prime}}=$ $s_{\tau(\lambda) \tau\left(\lambda^{\prime}\right)} s_{\lambda^{\prime}}^{\tau\left(\lambda^{\prime}\right)}$, which is a special case of (3.2).

We have thus, constructed all objects and proved all properties required by Theorem 1.7 with one exception, $\Lambda \times T$ need not be cofinite. We will obtain this last property by applying to the already obtained construction a known procedure which converts arbitrary systems to cofinite systems (see Lemma 6.31 of [4]).

STEP 8. Let $Z^{\star}=\left(Z_{\lambda}^{\star \alpha}, r_{\lambda \lambda^{\prime}}^{\star \alpha \alpha^{\prime}}, \Lambda \times T^{\star}\right)$ be a new rectangular system defined as follows. $T^{\star}$ is the set of all finite subsets $\alpha$ of $T$ (endowed with the ordering inherited from $T$ ) such that $\alpha$ has a terminal element $\bar{\alpha} \in T$. Because of anti-symmetry, $\bar{\alpha}$ is uniquely determined by $\alpha$. The ordering $\leq$ of $T^{\star}$ is given by the inclusion $\subseteq$. Clearly, $T^{\star}$ is cofinite. Note that $\alpha_{1} \leq \alpha_{2}$ implies $\bar{\alpha}_{1} \leq \bar{\alpha}_{2}$. Put $Z_{\lambda}^{\star \alpha}=Z_{\lambda}^{\bar{\alpha}}$ and $r_{\lambda \lambda^{\prime}}^{\star \alpha \alpha \alpha^{\prime}}=r_{\lambda \lambda^{\prime}}^{\bar{\alpha} \overline{\alpha^{\prime}}}$. Also define mappings $u_{\lambda}^{\star \alpha}: X_{\lambda} \rightarrow Z_{\lambda}^{\star \alpha}=Z_{\lambda}^{\bar{\alpha}}$ by putting $u_{\lambda}^{\star \alpha}=u_{\lambda}^{\bar{\alpha}}$. Note that the analogues of (1.2) and (1.3) hold. Indeed, (1.2) assumes the form $u_{\lambda}^{\star \alpha} p_{\lambda \lambda^{\prime}}=r_{\lambda \lambda^{\prime}}^{\star \alpha} u_{\lambda^{\prime}}^{\star \alpha}$, which is equivalent to $u_{\lambda}^{\bar{\alpha}} p_{\lambda \lambda^{\prime}}=r_{\lambda \lambda^{\prime}}^{\bar{\alpha}} u_{\lambda^{\prime}}^{\bar{\alpha}}$. Similarly, (1.3) assumes the form $r_{\lambda}^{\star \alpha \alpha^{\prime}} u_{\lambda}^{\star \alpha^{\prime}}=u_{\lambda}^{\star \alpha}$, which is equivalent to $r_{\lambda}^{\bar{\alpha} \overline{\alpha^{\prime}}} r_{\lambda}^{\overline{\alpha^{\prime}}}=u_{\lambda}^{\bar{\alpha}}$.

It remains to prove that the mapping $\boldsymbol{u}_{\lambda}^{\star}: X_{\lambda} \rightarrow Z_{\lambda}^{\star}=\left(Z_{\lambda}^{\star \alpha}, r_{\lambda}^{\star \alpha \alpha^{\prime}}, T^{\star}\right)$, formed by the mappings $u_{\lambda}^{\star \alpha}$, is a resolution. Consider the special mapping $\boldsymbol{f}=\left(f, f^{\alpha}\right): \boldsymbol{Z}_{\lambda} \rightarrow \boldsymbol{Z}_{\lambda}^{\star}$, where $f: T^{\star} \rightarrow T$ is given by $f(\alpha)=\bar{\alpha}$ and $f^{\alpha}: Z_{\lambda}^{f(\alpha)}=Z_{\lambda}^{\bar{\alpha}} \rightarrow Z_{\lambda}^{\bar{\alpha}}=Z_{\lambda}^{\star \alpha}$ is the identity mapping. That $\boldsymbol{f}: \boldsymbol{Z}_{\lambda} \rightarrow \boldsymbol{Z}_{\lambda}^{\star}$ is a special mapping follows from the fact that $f$ increases and $f^{\alpha} r_{\lambda \lambda^{\prime}}^{\bar{\alpha} \overline{\alpha^{\prime}}}=$ $r_{\lambda \lambda^{\prime}}^{\bar{\alpha} \overline{\alpha^{\prime}}}=r_{\lambda \lambda^{\prime}}^{\star \alpha \alpha^{\prime}} f^{\alpha^{\prime}}$. Moreover, $f$ is cofinal, because, for every $\tau \in T$, the element $\alpha=\{\tau\} \in T^{\star}$ has the property that $f(\alpha)=\overline{\{\tau\}}=\tau$. Note that the composition $\boldsymbol{f} \boldsymbol{u}_{\lambda}: X_{\lambda} \rightarrow \boldsymbol{Z}_{\lambda}^{\star}$ consists of mappings $f^{\alpha} u^{\bar{\alpha}}=u_{\lambda}^{\bar{\alpha}}=u_{\lambda}^{\star \alpha}$ and thus, coincides with $\boldsymbol{u}_{\lambda}^{\star}$. Since $\boldsymbol{u}_{\lambda}: X_{\lambda} \rightarrow \boldsymbol{Z}_{\lambda}$ is a resolution, an application of Lemma 2.1 yields the desired conclusion that also $\boldsymbol{u}_{\lambda}^{\star}$ is a resolution. 


\section{Proof of Theorem 1.7 in the Polyhedral Case}

The proof of Theorem 1.7 in the ANR case does not apply to the polyhedral case, because the Cartesian product of two (infinite) polyhedra need not be a polyhedron (CW-topology). Therefore, we give a different proof. It consists of a result of N. Uglešić (see[7], Theorem 2), here stated as Proposition 4.1, and of additional arguments, which we will describe in details and which are similar to some arguments used in the ANR case.

Proposition 4.1. Let $\boldsymbol{X}=\left(X_{\lambda}, p_{\lambda \lambda^{\prime}}, \Lambda\right)$ be a cofinite inverse system of topological spaces. Then there exist cofinite polyhedral resolutions $\boldsymbol{q}_{\lambda}: X_{\lambda} \rightarrow$ $\boldsymbol{Y}_{\lambda}, \lambda \in \Lambda$, and there exist mappings $\boldsymbol{v}_{\lambda \lambda^{\prime}}: \boldsymbol{Y}_{\lambda^{\prime}} \rightarrow \boldsymbol{Y}_{\lambda}, \lambda \leq \lambda^{\prime}$, such that $\boldsymbol{q}_{\lambda} p_{\lambda \lambda^{\prime}}=\boldsymbol{v}_{\lambda \lambda^{\prime}} \boldsymbol{q}_{\lambda^{\prime}}$. Moreover, $\boldsymbol{v}_{\lambda \lambda^{\prime}} \boldsymbol{v}_{\lambda^{\prime} \lambda^{\prime \prime}}=\boldsymbol{v}_{\lambda \lambda^{\prime \prime}}$, for $\lambda \leq \lambda^{\prime} \leq \lambda^{\prime \prime}$, and $\boldsymbol{v}_{\lambda \lambda}=\mathrm{id}$.

The assertion that the polyhedral resolutions $\boldsymbol{q}_{\lambda}: X_{\lambda} \rightarrow \boldsymbol{Y}_{\lambda}$ are cofinite is not included in the statement of Theorem 2 of [7]. However, this follows from the proof of that theorem (see e.g., Remark 2 and a remark in the proof of Lemma 2 of [7]). Let $\boldsymbol{Y}_{\lambda}=\left(Y_{\lambda}^{\mu}, q_{\lambda}^{\mu \mu^{\prime}}, M_{\lambda}\right)$ and let $\boldsymbol{q}_{\lambda}$ be given by mappings $q_{\lambda}^{\mu}: X_{\lambda} \rightarrow Y_{\lambda}^{\mu}, \mu \in M_{\lambda}$. Let the mappings $\boldsymbol{v}_{\lambda \lambda^{\prime}}$ be given by functions $v_{\lambda \lambda^{\prime}}: M_{\lambda} \rightarrow M_{\lambda^{\prime}}$ and by mappings $v_{\lambda \lambda^{\prime}}^{\mu}: Y_{\lambda^{\prime}}^{v_{\lambda \lambda^{\prime}}(\mu)} \rightarrow Y_{\lambda}^{\mu}$ such that, for $\mu, \mu^{\prime} \in M_{\lambda}, \mu \leq \mu^{\prime}$, there exists an index $\nu \in M_{\lambda^{\prime}}, \nu \geq v_{\lambda \lambda^{\prime}}(\mu), v_{\lambda \lambda^{\prime}}\left(\mu^{\prime}\right)$, for which

$$
v_{\lambda \lambda^{\prime}}^{\mu} q_{\lambda^{\prime}}^{v_{\lambda \lambda^{\prime}}(\mu) \nu}=q_{\lambda}^{\mu \mu^{\prime}} v_{\lambda \lambda^{\prime}}^{\mu^{\prime}} q_{\lambda^{\prime}}^{v_{\lambda \lambda^{\prime}}\left(\mu^{\prime}\right) \nu} .
$$

Note that whenever (4.1) holds for some $\nu$, then it also holds for every $\nu^{\prime} \geq \nu$. To see this it suffices to compose (4.1) on the right with $q_{\lambda^{\prime}}^{\nu \nu^{\prime}}$.

Following the proof of Theorem 3 in [7], we now consider the coproduct $M=\bigsqcup_{\lambda \in \Lambda} M_{\lambda}$ on which we define an ordering $\leq$ as follows. If $\mu \in M_{\lambda}$, $\mu^{\prime} \in M_{\lambda^{\prime}}$, we put $\mu \leq \mu^{\prime}$ provided $\lambda \leq \lambda^{\prime}$ and for every $\mu^{*} \in M_{\lambda}, \mu^{*} \leq \mu$, one has $v_{\lambda \lambda^{\prime}}\left(\mu^{*}\right) \leq \mu^{\prime}$ (in particular, $\left.v_{\lambda \lambda^{\prime}}(\mu) \leq \mu^{\prime}\right)$ and

$$
v_{\lambda \lambda^{\prime}}^{\mu^{*}} q_{\lambda^{\prime}}^{v_{\lambda \lambda^{\prime}}\left(\mu^{*}\right) \mu^{\prime}}=q_{\lambda}^{\mu^{*} \mu} v_{\lambda \lambda^{\prime}}^{\mu} q_{\lambda^{\prime}}^{v_{\lambda \lambda^{\prime}}(\mu) \mu^{\prime}} .
$$

If $\lambda=\lambda^{\prime}, \boldsymbol{v}_{\lambda \lambda^{\prime}}$ is the identity mapping and thus, condition $v_{\lambda \lambda^{\prime}}\left(\mu^{*}\right) \leq \mu^{\prime}$ becomes $\mu^{*} \leq \mu^{\prime}$ (in particular, $\mu \leq \mu^{\prime}$ ), while (4.1) assumes the form $q_{\lambda}^{\mu^{*} \mu^{\prime}}=$ $q_{\lambda}^{\mu^{*} \mu} q_{\lambda}^{\mu \mu^{\prime}}$, which always holds. Consequently, the new ordering, restricted to $M_{\lambda}$, coincides with the original ordering on $M_{\lambda}$. This implies antisymmetry of $\leq$ on $M$. Indeed, if $\mu \in M_{\lambda}, \mu^{\prime} \in M_{\lambda^{\prime}}, \mu \leq \mu^{\prime}$ and $\mu^{\prime} \leq \mu$, then $\lambda \leq \lambda^{\prime}$ and $\lambda^{\prime} \leq \lambda$ and thus, $\lambda=\lambda^{\prime}$. Since $\leq$ on $M_{\lambda}$ is antisymmetric, it follows that $\mu=\mu^{\prime}$. To prove transitivity of $\leq$, assume that $\mu \leq \mu^{\prime}, \mu^{\prime} \leq \mu^{\prime \prime}$ and $\mu^{*} \leq \mu$. Then the first inequality implies $v_{\lambda \lambda^{\prime}}\left(\mu^{*}\right) \leq \mu^{\prime}$ and (4.2). Since also $v_{\lambda \lambda^{\prime}}(\mu) \leq \mu^{\prime}$, the second inequality shows that $v_{\lambda^{\prime} \lambda^{\prime \prime}}\left(v_{\lambda \lambda^{\prime}}\left(\mu^{*}\right)\right) \leq \mu^{\prime \prime}$,

$$
v_{\lambda^{\prime} \lambda^{\prime \prime}}^{v_{\lambda \lambda^{\prime}}^{\prime}\left(\mu^{*}\right)} q_{\lambda^{\prime \prime}}^{v_{\lambda^{\prime} \lambda^{\prime \prime}}\left(v_{\lambda \lambda^{\prime}}\left(\mu^{*}\right)\right) \mu^{\prime \prime}}=q_{\lambda^{\prime}}^{v_{\lambda \lambda^{\prime}}}\left(\mu^{*}\right) \mu^{\prime} v_{\lambda^{\prime} \lambda^{\prime \prime}}^{\mu^{\prime}} q_{\lambda^{\prime \prime}}^{v_{\lambda^{\prime} \lambda^{\prime \prime}}}\left(\mu^{\prime}\right) \mu^{\prime \prime},
$$




$$
v_{\lambda^{\prime} \lambda^{\prime \prime}}^{v_{\lambda \lambda^{\prime}}^{\prime \prime}}(\mu) q_{\lambda^{\prime \prime}}^{v_{\lambda^{\prime} \lambda^{\prime \prime}}\left(v_{\lambda \lambda^{\prime}}(\mu)\right) \mu^{\prime \prime}}=q_{\lambda^{\prime}}^{v_{\lambda \lambda^{\prime}}}(\mu) \mu^{\prime} v_{\lambda^{\prime} \lambda^{\prime \prime}}^{\mu^{\prime}} q_{\lambda^{\prime \prime}}^{v_{\lambda^{\prime} \lambda^{\prime \prime}}}\left(\mu^{\prime}\right) \mu^{\prime \prime} .
$$

Note that $\boldsymbol{v}_{\lambda \lambda^{\prime}} \boldsymbol{v}_{\lambda^{\prime} \lambda^{\prime \prime}}=\boldsymbol{v}_{\lambda \lambda^{\prime \prime}}$ implies $v_{\lambda^{\prime} \lambda^{\prime \prime}} v_{\lambda \lambda^{\prime}}=v_{\lambda \lambda^{\prime \prime}}, v_{\lambda \lambda^{\prime}}^{\mu^{*}} v_{\lambda^{\prime} \lambda^{\prime \prime}}^{v_{\lambda^{\prime}}\left(\mu^{*}\right)}=v_{\lambda \lambda^{\prime \prime}}^{\mu^{*}}$ and $v_{\lambda \lambda^{\prime}}^{\mu} v_{\lambda^{\prime} \lambda^{\prime \prime}}^{v_{\lambda \lambda^{\prime}}}(\mu)=v_{\lambda \lambda^{\prime \prime}}^{\mu}$. Therefore, $v_{\lambda \lambda^{\prime \prime}}\left(\mu^{*}\right) \leq \mu^{\prime \prime}$ and, by (4.3), (4.2) and (4.4),

$$
v_{\lambda \lambda^{\prime \prime}}^{\mu^{*}} q_{\lambda^{\prime \prime}}^{v_{\lambda \lambda^{\prime \prime}}\left(\mu^{*}\right) \mu^{\prime \prime}}=q_{\lambda}^{\mu^{*} \mu} v_{\lambda \lambda^{\prime \prime}}^{\mu} q_{\lambda^{\prime \prime}}^{v_{\lambda \lambda^{\prime \prime}}(\mu) \mu^{\prime \prime}}
$$

and thus, $\mu \leq \mu^{\prime \prime}$.

In the sequel we will need the following property of $M$ : If $\mu \in M_{\lambda}$ and $\lambda \leq \lambda^{\prime}$, then there exists a $\mu^{\prime} \in M_{\lambda^{\prime}}$ such that $\mu \leq \mu^{\prime}$. Indeed, since $M_{\lambda}$ is cofinite, the set of all predecessors of $\mu$ in $M_{\lambda}$ is a finite set $\left\{\mu_{1}^{*}, \ldots \mu_{k}^{*}\right\}$. By property (4.1) of $\boldsymbol{v}_{\lambda \lambda^{\prime}}$, for every $1 \leq i \leq k$, there is a $\nu_{i} \in M_{\lambda^{\prime}}$ such that, for $1 \leq i \leq k, \gamma_{\lambda \lambda^{\prime}}\left(\mu_{i}^{*}\right), \gamma_{\lambda \lambda^{\prime}}(\mu) \leq \nu_{i}$ and

$$
v_{\lambda \lambda^{\prime}}^{\mu_{i}^{*}} q_{\lambda^{\prime}}^{\gamma_{\lambda \lambda^{\prime}}\left(\mu_{i}^{*}\right) \nu_{i}}=q_{\lambda}^{\mu_{i}^{*} \mu} v_{\lambda \lambda^{\prime}}^{\mu} q_{\lambda^{\prime}}^{\gamma_{\lambda \lambda^{\prime}}(\mu) \nu_{i}}
$$

Choosing for $\mu^{\prime}$ an element of $M_{\lambda^{\prime}}$ such that $\nu_{1}, \ldots, \nu_{k} \leq \mu^{\prime}$, one concludes that, for all predecessors $\mu_{i}^{*}$ of $\mu, v_{\lambda \lambda^{\prime}}\left(\mu_{i}^{*}\right) \leq \mu^{\prime}$ and also

$$
v_{\lambda \lambda^{\prime}}^{\mu_{i}^{*}} q_{\lambda^{\prime}}^{v_{\lambda \lambda^{\prime}}}\left(\mu_{i}^{*}\right) \mu^{\prime}=q_{\lambda}^{\mu_{i}^{*} \mu} v_{\lambda \lambda^{\prime}}^{\mu} q_{\lambda^{\prime}}^{v_{\lambda \lambda^{\prime}}(\mu) \mu^{\prime}} .
$$

By definition, this means that $\mu \leq \mu^{\prime}$.

We now define a new ordered set $T$ as follows. Let $\tilde{T}$ be the set of all functions $\tilde{\tau}: \Lambda \rightarrow M$ such that $\tilde{\tau}(\lambda) \in M_{\lambda}$. Order $\tilde{T}$ by putting $\tilde{\tau} \leq \tilde{\tau}^{\prime}$ provided $\tilde{\tau}(\lambda) \leq \tilde{\tau}^{\prime}(\lambda)$, for all $\lambda \in \Lambda$. Clearly, $\tilde{T}$ is directed. Let $T$ be the subset of $\tilde{T}$ consisting of all increasing functions $\tau: \Lambda \rightarrow M$.

For any function $\tilde{\tau} \in \tilde{T}$, there exists a function $\tau \in T$ such that $\tilde{\tau} \leq \tau$. We define the values $\tau(\lambda)$, using induction by the number of predecessors of $\lambda$. If this number is 1 , we put $\tau(\lambda)=\tilde{\tau}(\lambda)$. If we have already defined values $\tau\left(\lambda_{i}\right) \in M_{\lambda_{i}}, \tau\left(\lambda_{i}\right) \leq \tilde{\tau}\left(\lambda_{i}\right)$, for all predecessors $\lambda_{1}, \lambda_{2}, \ldots, \lambda_{k}$ of $\lambda$, different from $\lambda$, we choose (using the above stated property of $M$ ) elements $\mu_{i} \in M_{\lambda}$ such that $\tau\left(\lambda_{i}\right) \leq \mu_{i}$. Then we take for $\tau(\lambda)$ any element $\mu \in M_{\lambda}$ such that $\mu \geq \mu_{1}, \ldots, \mu_{k}, \tilde{\tau}(\lambda)$. By the transitivity of $\leq, \tau\left(\lambda_{i}\right) \leq \tau(\lambda)$ and $\tilde{\tau}(\lambda) \leq \tau(\lambda)$. The first property implies that $\tau$ is an increasing functions from $\tilde{T}$, i.e., $\tau \in T$, and the second property implies that $\tilde{\tau} \leq \tau$. It is now easy to see that $T$ is a directed set. Indeed, let $\tau_{1}, \tau_{2} \in T \subseteq \tilde{T}$. Clearly, there exists a function $\tilde{\tau} \in \tilde{T}$ such that $\tau_{1}, \tau_{2} \leq \tilde{\tau}$. If we choose $\tau \in T$ such that $\tilde{\tau} \leq \tau$, then $\tau_{1}, \tau_{2} \leq \tau$.

We will now define a rectangular system of polyhedra $\boldsymbol{Z}=\left(Z_{\lambda}^{\tau}, r_{\lambda \lambda^{\prime}}^{\tau \tau^{\prime}}, \Lambda \times\right.$ $T)$. For $\lambda \in \Lambda, \tau \in T$, put $Z_{\lambda}^{\tau}=Y_{\lambda}^{\tau(\lambda)}$. For $\lambda \leq \lambda^{\prime}, \tau \in T$, we define $r_{\lambda \lambda^{\prime}}^{\tau}: Z_{\lambda^{\prime}}^{\tau} \rightarrow Z_{\lambda}^{\tau}$ by putting

$$
r_{\lambda \lambda^{\prime}}^{\tau}=v_{\lambda \lambda^{\prime}}^{\tau(\lambda)} q_{\lambda^{\prime}}^{v_{\lambda \lambda^{\prime}}(\tau(\lambda)) \tau\left(\lambda^{\prime}\right)}
$$

i.e., by taking for $r_{\lambda \lambda^{\prime}}^{\tau}$ the composition of the mappings $q_{\lambda^{\lambda^{\prime}}}^{v_{\lambda \lambda^{\prime}}(\tau(\lambda)) \tau\left(\lambda^{\prime}\right)}: Z_{\lambda^{\prime}}^{\tau}=$ $Y_{\lambda^{\prime}}^{\tau\left(\lambda^{\prime}\right)} \rightarrow Y_{\lambda^{\prime}}^{v_{\lambda \lambda^{\prime}}(\tau(\lambda))}$ and $v_{\lambda \lambda^{\prime}}^{\tau(\lambda)}: Y_{\lambda^{\prime}}^{\gamma_{\lambda \lambda^{\prime}}(\tau(\lambda))} \rightarrow Y_{\lambda}^{\tau(\lambda)}=Z_{\lambda}^{\tau}$. Note that $r_{\lambda \lambda^{\prime}}^{\tau}$ 
is well defined because $\tau$ is an increasing function and thus, $\lambda \leq \lambda^{\prime}$ implies $\tau(\lambda) \leq \tau\left(\lambda^{\prime}\right), \tau(\lambda) \in M_{\lambda}$ and $\tau\left(\lambda^{\prime}\right) \in M_{\lambda^{\prime}}$. However, by the definition of the ordering $\leq$ in $M$, the latter inequality implies $v_{\lambda \lambda^{\prime}}(\tau(\lambda)) \leq \tau\left(\lambda^{\prime}\right)$. For $\lambda \in \Lambda$ and $\tau \leq \tau^{\prime}$ we define $r_{\lambda}^{\tau \tau^{\prime}}: Z_{\lambda}^{\tau^{\prime}} \rightarrow Z_{\lambda}^{\tau}$, by putting

$$
r_{\lambda}^{\tau \tau^{\prime}}=q_{\lambda}^{\tau(\lambda) \tau^{\prime}(\lambda)} .
$$

Let us show that, for $\lambda \leq \lambda^{\prime} \leq \lambda^{\prime \prime}, \tau \in T$, one has

$$
r_{\lambda \lambda^{\prime}}^{\tau} r_{\lambda^{\prime} \lambda^{\prime \prime}}^{\tau}=r_{\lambda \lambda^{\prime \prime}}^{\tau}
$$

Indeed, by (4.8),

$$
r_{\lambda \lambda^{\prime}}^{\tau} r_{\lambda^{\prime} \lambda^{\prime \prime}}^{\tau}=v_{\lambda \lambda^{\prime}}^{\tau(\lambda)} q_{\lambda^{\prime}}^{v_{\lambda \lambda^{\prime}}(\tau(\lambda)) \tau\left(\lambda^{\prime}\right)} v_{\lambda^{\prime} \lambda^{\prime \prime}}^{\tau\left(\lambda^{\prime}\right)} q_{\lambda^{\prime \prime}}^{v_{\lambda^{\prime} \lambda^{\prime \prime}}\left(\tau\left(\lambda^{\prime}\right)\right) \tau\left(\lambda^{\prime \prime}\right)} .
$$

Moreover, since $v_{\lambda \lambda^{\prime}}(\tau(\lambda)) \leq \tau\left(\lambda^{\prime}\right)$ and $\tau\left(\lambda^{\prime}\right) \leq \tau\left(\lambda^{\prime \prime}\right)$, (4.2) yields

$$
q_{\lambda^{\prime}}^{v_{\lambda \lambda^{\prime}}}(\tau(\lambda)) \tau\left(\lambda^{\prime}\right) v_{\lambda^{\prime} \lambda^{\prime \prime}}^{\tau\left(\lambda^{\prime}\right)} q_{\lambda^{\prime \prime}}^{v_{\lambda^{\prime} \lambda^{\prime \prime}}\left(\tau\left(\lambda^{\prime}\right)\right) \tau\left(\lambda^{\prime \prime}\right)}=v_{\lambda^{\prime} \lambda^{\prime \prime}}^{v_{\lambda \lambda^{\prime \prime}}(\tau(\lambda))} q_{\lambda^{\prime \prime}}^{v_{\lambda \lambda^{\prime \prime}}(\tau(\lambda)) \tau\left(\lambda^{\prime \prime}\right)} .
$$

Consequently,

$$
r_{\lambda \lambda^{\prime}}^{\tau} r_{\lambda^{\prime} \lambda^{\prime \prime}}^{\tau}=v_{\lambda \lambda^{\prime}}^{\tau(\lambda)} v_{\lambda^{\prime} \lambda^{\prime \prime}}^{v_{\lambda \lambda^{\prime}}^{\prime}(\tau(\lambda))} q_{\lambda^{\prime \prime}}^{v_{\lambda \lambda^{\prime \prime}}(\tau(\lambda)) \tau\left(\lambda^{\prime \prime}\right)}=v_{\lambda \lambda^{\prime \prime}}^{\tau(\lambda)} q_{\lambda^{\prime \prime}}^{v_{\lambda \lambda^{\prime \prime}}}(\tau(\lambda)) \tau\left(\lambda^{\prime \prime}\right)=r_{\lambda \lambda^{\prime \prime}}^{\tau}
$$

The verification that $\lambda \in \Lambda$ and $\tau \leq \tau^{\prime} \leq \tau^{\prime \prime}$ yields

$$
r_{\lambda}^{\tau \tau^{\prime}} r_{\lambda}^{\tau^{\prime} \tau^{\prime \prime}}=r_{\lambda}^{\tau \tau^{\prime \prime}}
$$

is straightforward. In order to complete the proof that $Z$ is a rectangular inverse system, it remains to show that

$$
r_{\lambda \lambda^{\prime}}^{\tau} r_{\lambda^{\prime}}^{\tau \tau^{\prime}}=r_{\lambda}^{\tau \tau^{\prime}} r_{\lambda \lambda^{\prime}}^{\tau^{\prime}}, \lambda \leq \lambda^{\prime}, \tau \leq \tau^{\prime},
$$

First note that $\tau \leq \tau^{\prime}$ implies $\tau(\lambda) \leq \tau^{\prime}(\lambda)$. Therefore, by $\tau^{\prime}(\lambda) \leq \tau^{\prime}\left(\lambda^{\prime}\right)$ and by the definition of the ordering $\leq$,

$$
v_{\lambda \lambda^{\prime}}^{\tau(\lambda)} q_{\lambda^{\prime}}^{v_{\lambda \lambda^{\prime}}}(\tau(\lambda)) \tau^{\prime}\left(\lambda^{\prime}\right)=q_{\lambda}^{\tau(\lambda) \tau^{\prime}(\lambda)} v_{\lambda \lambda^{\prime}}^{\tau^{\prime}(\lambda)} q_{\lambda^{\prime}}^{v_{\lambda \lambda^{\prime}}\left(\tau^{\prime}(\lambda)\right) \tau^{\prime}\left(\lambda^{\prime}\right)} .
$$

(4.16) yields (4.15) if one takes into account that $r_{\lambda \lambda^{\prime}}^{\tau}=v_{\lambda \lambda^{\prime}}^{\tau(\lambda)} q_{\lambda^{\prime}}^{v_{\lambda \lambda^{\prime}}(\tau(\lambda)) \tau\left(\lambda^{\prime}\right)}$, $r_{\lambda \lambda^{\prime}}^{\tau^{\prime}}=v_{\lambda \lambda^{\prime}}^{\tau^{\prime}(\lambda)} q_{\lambda^{\prime}}^{v_{\lambda \lambda^{\prime}}\left(\tau^{\prime}(\lambda)\right) \tau^{\prime}\left(\lambda^{\prime}\right)}$ and $q_{\lambda^{\prime}}^{v_{\lambda \lambda^{\prime}}(\tau(\lambda)) \tau^{\prime}\left(\lambda^{\prime}\right)}=q_{\lambda^{\prime}}^{v_{\lambda \lambda^{\prime}}}(\tau(\lambda)) \tau\left(\lambda^{\prime}\right) q_{\lambda^{\prime}}^{\tau\left(\lambda^{\prime}\right) \tau^{\prime}\left(\lambda^{\prime}\right)}$.

For a fixed $\lambda \in \Lambda$, consider the resolution $\boldsymbol{q}_{\lambda}: X_{\lambda} \rightarrow \boldsymbol{Y}_{\lambda}$. Also consider the function $f: T \rightarrow M_{\lambda}$, defined by $f(\tau)=\tau(\lambda)$. This function is increasing, because $\tau \leq \tau^{\prime}$ implies $\tau(\lambda) \leq \tau^{\prime}(\lambda)$. Moreover, $f$ is cofinal. Indeed, for an arbitrary element $\mu \in M_{\lambda}$, we first define a function $\tilde{\tau} \in \tilde{T}$, by putting $\tilde{\tau}(\lambda)=\mu$ and by taking for $\tilde{\tau}\left(\lambda^{\prime}\right), \lambda^{\prime} \neq \lambda$, any value in $M_{\lambda^{\prime}}$. Then we choose for $\tau$ a function from $T$ such that $\tilde{\tau} \leq \tau$. Clearly, $f(\tau)=\tau(\lambda) \geq \tilde{\tau}(\lambda)=\mu$.

Now consider the special mapping $\boldsymbol{f}=\left(f, f^{\tau}\right): \boldsymbol{Y}_{\lambda} \rightarrow \boldsymbol{Z}_{\lambda}=\left(Z_{\lambda}^{\tau}, r_{\lambda}^{\tau \tau^{\prime}}, T\right)$, where $f^{\tau}: Y_{\lambda}^{f(\tau)}=Y_{\lambda}^{\tau(\lambda)} \rightarrow Y_{\lambda}^{\tau(\lambda)}=Z_{\lambda}^{\tau}$ is the identity mapping. That $f$ is indeed a special mapping follows from the equality $r_{\lambda}^{\tau \tau^{\prime}} f^{\tau^{\prime}}=q_{\lambda}^{\tau(\lambda) \tau^{\prime}(\lambda)}=$ $f^{\tau} q_{\lambda}^{\tau(\lambda) \tau^{\prime}(\lambda)}$. The composition $\boldsymbol{u}_{\lambda}=\boldsymbol{f} \boldsymbol{q}_{\lambda}: X_{\lambda} \rightarrow \boldsymbol{Z}_{\lambda}$ consists of mappings 
$u_{\lambda}^{\tau}=f^{\tau} q_{\lambda}^{f(\tau)}=q_{\lambda}^{\tau(\lambda)}: X_{\lambda} \rightarrow Y_{\lambda}^{\tau(\lambda)}=Z_{\lambda}^{\tau}$. An application of Lemma 2.1 shows that $\boldsymbol{u}_{\lambda}: X_{\lambda} \rightarrow \boldsymbol{Z}_{\lambda}$ is a resolution.

Now let us prove that $\boldsymbol{u}_{\lambda} p_{\lambda \lambda^{\prime}}=\boldsymbol{r}_{\lambda \lambda^{\prime}} \boldsymbol{u}_{\lambda^{\prime}}$, i.e., $u_{\lambda}^{\tau} p_{\lambda \lambda^{\prime}}=r_{\lambda \lambda^{\prime}}^{\tau} u_{\lambda^{\prime}}^{\tau}$. Indeed, $\boldsymbol{q}_{\lambda} p_{\lambda \lambda^{\prime}}=\boldsymbol{v}_{\lambda \lambda^{\prime}} \boldsymbol{q}_{\lambda^{\prime}}$ implies $q_{\lambda}^{\tau(\lambda)} p_{\lambda \lambda^{\prime}}=v_{\lambda \lambda^{\prime}}^{\tau(\lambda)} q_{\lambda^{\prime}}^{v_{\lambda \lambda^{\prime}}(\tau(\lambda))}$. Since

$$
q_{\lambda^{\prime}}^{v_{\lambda \lambda^{\prime}}(\tau(\lambda))}=q_{\lambda^{\prime}}^{v_{\lambda \lambda^{\prime}}(\tau(\lambda)) \tau\left(\lambda^{\prime}\right)} q_{\lambda^{\prime}}^{\tau\left(\lambda^{\prime}\right)}
$$

and $r_{\lambda \lambda^{\prime}}^{\tau}$ is given by (4.8), we see that $u_{\lambda}^{\tau} p_{\lambda \lambda^{\prime}}=q_{\lambda}^{\tau(\lambda)} p_{\lambda \lambda^{\prime}}=v_{\lambda \lambda^{\prime}}^{\tau(\lambda)} q_{\lambda^{\prime}}^{v_{\lambda \lambda^{\prime}}(\tau(\lambda))}=$ $v_{\lambda \lambda^{\prime}}^{\tau(\lambda)} q_{\lambda^{\prime}}^{v_{\lambda \lambda^{\prime}}(\tau(\lambda)) \tau\left(\lambda^{\prime}\right)} q_{\lambda^{\prime}}^{\tau\left(\lambda^{\prime}\right)}=r_{\lambda \lambda^{\prime}}^{\tau} u_{\lambda^{\prime}}^{\tau}$.

In this way we have proved all the assertions of Theorem 1.7 in the polyhedral case, except for the cofiniteness of $T$. To obtain also this property, it suffices to repeat Step 8 from the proof of Theorem 1.7 in the ANR case.

\section{Proofs of Other Results}

Proof of TheOrem 1.9. We need to prove that $\boldsymbol{v}$ is a resolution of $X$, i.e., it has properties (B1) and (B2). To establish (B1), assume that $\mathcal{U}$ is a normal covering of $X$. Then, by property (B1) for $\boldsymbol{p}$, there exists a $\lambda \in \Lambda$ and a normal covering $\mathcal{U}_{\lambda}$ of $X_{\lambda}$ such that $p_{\lambda}^{-1}\left(\mathcal{U}_{\lambda}\right)$ refines $\mathcal{U}$. Using (B1) for $\boldsymbol{u}_{\lambda}$, we conclude that there exist a $\tau \in T$ and a normal covering $\mathcal{U}_{\lambda}^{\tau}$ of $Z_{\lambda}^{\tau}$ such that $\left(u_{\lambda}^{\tau}\right)^{-1}\left(\mathcal{U}_{\lambda}^{\tau}\right)$ refines $\mathcal{U}_{\lambda}$. Since $v_{\lambda}^{\tau}=u_{\lambda}^{\tau} p_{\lambda}$, it follows that $\left(v_{\lambda}^{\tau}\right)^{-1}\left(\mathcal{U}_{\lambda}^{\tau}\right)$ refines $\mathcal{U}$.

To establish (B2) for $\boldsymbol{v}$, assume that $\mathcal{U}_{\lambda}^{\tau}$ is a normal covering of $Z_{\lambda}^{\tau}$ and let $\mathcal{V}_{\lambda}^{\tau}$ be a normal star-refinement of $\mathcal{U}_{\lambda}^{\tau}$. Consider the normal covering $\mathcal{V}_{\lambda}=\left(u_{\lambda}^{\tau}\right)^{-1}\left(\mathcal{V}_{\lambda}^{\tau}\right)$ of $X_{\lambda}$. By (B2) for $\boldsymbol{p}$, there exists a $\lambda^{\prime} \geq \lambda$ such that

$$
p_{\lambda \lambda^{\prime}}\left(X_{\lambda^{\prime}}\right) \subseteq \operatorname{St}\left(\overline{p_{\lambda}(X)}, \mathcal{V}_{\lambda}\right) \text {. }
$$

We claim that

$$
u_{\lambda}^{\tau} p_{\lambda \lambda^{\prime}}\left(X_{\lambda^{\prime}}\right) \subseteq \operatorname{St}\left(\overline{v_{\lambda}^{\tau}(X)}, \mathcal{V}_{\lambda}^{\tau}\right)
$$

Indeed, by (5.1), for every $x \in X_{\lambda^{\prime}}$, there exists a member $V_{\lambda}$ of $\mathcal{V}_{\lambda}$ and a point $y \in V_{\lambda} \cap \overline{p_{\lambda}(X)}$ such that $p_{\lambda \lambda^{\prime}}(x) \in V_{\lambda}$. Moreover, there exists a member $V \in \mathcal{V}_{\lambda}^{\tau}$ such that $\left(u_{\lambda}^{\tau}\right)^{-1}(V)=V_{\lambda}$. Consequently, $u_{\lambda}^{\tau} p_{\lambda \lambda^{\prime}}(x) \in V$, $u_{\lambda}^{\tau}(y) \in V$ and $\left.u_{\lambda}^{\tau}(y) \in u_{\lambda}^{\tau} \overline{\left(p_{\lambda}(X)\right.}\right) \subseteq \overline{u_{\lambda}^{\tau} p_{\lambda}(X)}=\overline{v_{\lambda}^{\tau}(X)}$. The last two relations show that $V \subseteq \operatorname{St}\left(\overline{v_{\lambda}^{\tau}(X)}, \mathcal{V}_{\lambda}^{\tau}\right)$ and thus, $u_{\lambda}^{\tau} p_{\lambda \lambda^{\prime}}(x) \in \operatorname{St}\left(\overline{v_{\lambda}^{\tau}(X)}, \mathcal{V}_{\lambda}^{\tau}\right)$, which establishes (5.2). By (1.2), $u_{\lambda}^{\tau} p_{\lambda \lambda^{\prime}}=r_{\lambda \lambda^{\prime}}^{\tau} u_{\lambda^{\prime}}^{\tau}$ and thus, (5.2) becomes

$$
r_{\lambda \lambda^{\prime}}^{\tau} u_{\lambda^{\prime}}^{\tau}\left(X_{\lambda^{\prime}}\right) \subseteq \operatorname{St}\left(\overline{v_{\lambda}^{\tau}(X)}, \mathcal{V}_{\lambda}^{\tau}\right)
$$

Now consider the normal covering $\mathcal{V}_{\lambda^{\prime}}^{\tau}=\left(r_{\lambda \lambda^{\prime}}^{\tau}\right)^{-1}\left(\mathcal{V}_{\lambda}^{\tau}\right)$. We claim that

$$
r_{\lambda \lambda^{\prime}}^{\tau}\left(\operatorname{St}\left(\overline{u_{\lambda^{\prime}}^{\tau}\left(X_{\lambda^{\prime}}\right)}\right), \mathcal{V}_{\lambda^{\prime}}^{\tau}\right) \subseteq \operatorname{St}\left(\overline{v_{\lambda}^{\tau}(X)}, \mathcal{U}_{\lambda}^{\tau}\right) .
$$

Indeed, if $y \in \operatorname{St}\left(\overline{u_{\lambda^{\prime}}^{\tau}\left(X_{\lambda^{\prime}}\right)}, \mathcal{V}_{\lambda^{\prime}}^{\tau}\right)$, then there exists a member $V^{\prime}$ of $\mathcal{V}_{\lambda^{\prime}}^{\tau}$ such that $y \in V^{\prime}$ and $V^{\prime} \cap \overline{u_{\lambda^{\prime}}^{\tau}\left(X_{\lambda^{\prime}}\right)} \neq \emptyset$. Since $V^{\prime}$ is open, it follows that also $V^{\prime} \cap u_{\lambda^{\prime}}^{\tau}\left(X_{\lambda^{\prime}}\right) \neq \emptyset$ and thus, $r_{\lambda \lambda^{\prime}}^{\tau}\left(V^{\prime}\right) \cap r_{\lambda \lambda^{\prime}}^{\tau} u_{\lambda^{\prime}}^{\tau}\left(X_{\lambda^{\prime}}\right) \neq \emptyset$. By the definition 
of $\mathcal{V}_{\lambda^{\prime}}^{\tau}$, there exists a member $W$ of $\mathcal{V}_{\lambda}^{\tau}$ such that $V^{\prime}=\left(r_{\lambda \lambda^{\prime}}^{\tau}\right)^{-1}(W)$ and thus, $W \cap r_{\lambda \lambda^{\prime}}^{\tau} u_{\lambda^{\prime}}^{\tau}\left(X_{\lambda^{\prime}}\right) \neq \emptyset$. Now (5.3) implies that $W \cap \operatorname{St}\left(\overline{v_{\lambda}^{\tau}(X)}, \mathcal{V}_{\lambda}^{\tau}\right) \neq$ $\emptyset$. Consequently, there is a member $W^{\prime}$ of $\mathcal{V}_{\lambda}^{\tau}$ such that $W \cap W^{\prime} \neq \emptyset$ and $W^{\prime} \cap \overline{v_{\lambda}^{\tau}(X)} \neq \emptyset$. Since $\mathcal{V}_{\lambda}^{\tau}$ is a star-refinement of $\mathcal{U}_{\lambda}^{\tau}$, there exists a member $U$ of $\mathcal{U}_{\lambda}^{\tau}$ such that $W \cup W^{\prime} \subseteq U$. Clearly, $U \cap \overline{v_{\lambda}^{\tau}(X)} \neq \emptyset$ and thus, $r_{\lambda \lambda^{\prime}}^{\tau}(y) \in$ $r_{\lambda \lambda^{\prime}}^{\tau}\left(V^{\prime}\right) \subseteq W \subseteq U \subseteq \operatorname{St}\left(\overline{v_{\lambda}^{\tau}(X)}, \mathcal{U}_{\lambda}^{\tau}\right)$, which establishes (5.4).

By property (B2) for the resolution $\boldsymbol{u}_{\lambda^{\prime}}: X_{\lambda^{\prime}} \rightarrow \boldsymbol{Z}_{\lambda^{\prime}}$, there exists a $\tau^{\prime} \geq$ $\tau$ such that $r_{\lambda^{\prime}}^{\tau \tau^{\prime}}\left(Z_{\lambda^{\prime}}^{\tau^{\prime}}\right) \subseteq \operatorname{St}\left(\overline{u_{\lambda^{\prime}}^{\tau}\left(X_{\lambda^{\prime}}\right)}, \mathcal{V}_{\lambda^{\prime}}^{\tau}\right)$ and thus, by $(5.4), r_{\lambda \lambda^{\prime}}^{\tau \tau^{\prime}}\left(Z_{\lambda^{\prime}}^{\tau^{\prime}}\right)=$ $r_{\lambda \lambda^{\prime}}^{\tau} r_{\lambda^{\prime}}^{\tau \tau^{\prime}}\left(Z_{\lambda^{\prime}}^{\tau^{\prime}}\right) \subseteq r_{\lambda \lambda^{\prime}}^{\tau}\left(\mathrm{St}\left(\overline{u_{\lambda^{\prime}}^{\tau}\left(X_{\lambda^{\prime}}\right)}, \mathcal{V}_{\lambda^{\prime}}^{\tau}\right)\right) \subseteq \mathrm{St}\left(\overline{v_{\lambda}^{\tau}(X)}, \mathcal{U}_{\lambda}^{\tau}\right)$, which shows that $\boldsymbol{v}$ also has property (B2).

Proof of Theorems 1.1 And 1.5. A proof of Theorem 1.1 is obtained by a small variation of the proof of Theorem 1.7 in the ANR case. All spaces which appear in the proof are compact Hausdorff spaces. Therefore, resolutions and limits coincide. Whenever in the proof of Theorem 1.7 appears an inverse systems of ANRs, at the corresponding place in the proof of Theorem 1.1 we have a system of compact polyhedra (which are always compact ANRs). In Step 4, instead of considering open neighborhoods $G$ of $\overline{q_{\lambda}^{\mu}\left(X_{\lambda}\right)}=q_{\lambda}^{\mu}\left(X_{\lambda}\right)$ in $Y_{\lambda}^{\mu}$, we consider neighborhoods of $q_{\lambda}^{\mu}\left(X_{\lambda}\right)$ in $Y_{\lambda}^{\mu}$, which are compact polyhedra. Another proof is obtained from the proof of Theorem 1.7 in the polyhedral case. Indeed, in the proof of Proposition 4.1 (see [7]) all members $Y_{\lambda}^{\mu}$ of $\boldsymbol{Y}_{\lambda}$ are either nerves of open coverings of $X_{\lambda}$ or subcomplexes of their subdivisions. In the compact case, it suffices to consider finite open coverings. Consequently, one can assume that all $Y_{\lambda}^{\mu}$, hence also all $Z_{\lambda}^{\tau}=Y_{\lambda}^{\tau(\lambda)}$, are compact polyhedra.

Theorem 1.5 immediately follows from Theorem 1.9. Indeed, if $\boldsymbol{p}$ is a limit, then it is also a resolution. Consequently, $\boldsymbol{v}$ is also a resolution. However, since we are in the compact situation, this resolution is actually a limit.

The proof of Corollary 1.3 uses the following elementary lemma on directed sets.

Lemma 5.1. In a directed set $M$ every countable subset $M^{\prime} \subseteq M$ is contained in a countable directed subset $M_{1} \subseteq M$. More precisely, if $M^{\prime}=\left\{\mu_{1}, \mu_{2}, \ldots\right\}$, then there exists an increasing sequence $m_{1} \leq m_{2} \leq \ldots$ of elements of $M$ such that $\mu_{i} \leq m_{i}$, for $i \in \mathbb{N}$, and thus the set $M_{1}=$ $M^{\prime} \cup\left\{m_{1}, m_{2}, \ldots\right\}$ is a countable directed subset of $M$, which contains the set $M^{\prime}$ and the set $\left\{m_{1} \leq m_{2} \leq \ldots\right\}$ is cofinal in $M_{1}$.

Proof. We define the sequence $m_{i}$ by induction, beginning with $m_{1}=$ $\mu_{1}$. Assume that we have already defined $m_{1} \leq m_{2} \leq \ldots \leq m_{i}$ in such a way that $\mu_{j} \leq m_{j}$, for $1 \leq j \leq i$. We take for $m_{i+1}$ an element of $M$ such that $m_{i+1} \geq m_{i}, \mu_{i+1}$. 
Proof of Corollary 1.3. Let $\boldsymbol{X}=\left(X_{n}, p_{n n^{\prime}}, \mathbb{N}\right)$ be an inverse sequence of metric compacta. By Theorem 1.1, there exists a rectangular inverse system of compact polyhedra $\boldsymbol{Z}=\left(Z_{n}^{\mu}, r_{n n^{\prime}}^{\mu \mu^{\prime}}, \mathbb{N} \times M\right)$ and there exist limits $\boldsymbol{u}_{n}=\left(u_{n}^{\mu}\right): X_{n} \rightarrow \boldsymbol{Z}_{n}$ of the vertical systems $\boldsymbol{Z}_{n}=\left(Z_{n}^{\mu}, r_{n}^{\mu \mu^{\prime}}, M\right)$ such that $u_{n}^{\mu} p_{n n^{\prime}}=r_{n n^{\prime}}^{\mu} u_{n^{\prime}}^{\mu}$, for $n \leq n^{\prime}$ and $\mu \in M$. Since $X_{n}$ is a metrizable compactum, there exists a countable collection of open coverings $\left\{\mathcal{U}_{n}^{i} \mid i \in \mathbb{N}\right\}$ of $X_{n}$, which form a basis of open coverings of $X_{n}$, i.e., for every open covering $\mathcal{U}_{n}$ of $X_{n}$, there exists an $i \in \mathbb{N}$ such that $\mathcal{U}_{n}^{i}$ refines $\mathcal{U}_{n}$. By property (B1) of $\boldsymbol{u}_{n}$, for every $i \in \mathbb{N}$, there is a $\mu_{n}^{i} \in M$ and there is an open covering $\mathcal{V}_{n}^{i}$ of $Z_{n}^{\mu_{n}^{i}}$ such that $\left(u_{n}^{\mu_{n}^{i}}\right)^{-1}\left(\mathcal{V}_{n}^{i}\right)$ refines $\mathcal{U}_{n}^{i}$. Clearly, $M^{\prime}=\left\{\mu_{n}^{i} \mid(n, i) \in \mathbb{N} \times \mathbb{N}\right\}$ is a countable subset of $M$ such that for every directed subset $M^{\prime \prime} \subseteq M$, which contains $M^{\prime}$, and every $n \in \mathbb{N}$, the restriction $\boldsymbol{u}_{n}\left|M^{\prime \prime}: X_{n} \rightarrow \boldsymbol{Z}_{n}\right| M^{\prime \prime}$ has property (B1). By Lemma 5.1, there exists a countable directed subset $M_{1} \subseteq M$ such that $M^{\prime} \subseteq M_{1}$.

We will now define by induction a sequence of countable directed subsets $M_{1} \subseteq M_{2} \subseteq \ldots$ of $M$ such that, for every $\mu \in M_{k}$, every $n \in \mathbb{N}$ and every open neighborhood $V_{n}^{\mu}$ of $r_{n}^{\mu}\left(X_{n}\right)$ in $Z_{n}^{\mu}$, there is a $\mu^{\prime} \in M_{k+1}, \mu^{\prime} \geq \mu$, such that $r_{n}^{\mu \mu^{\prime}}\left(Z_{n}^{\mu^{\prime}}\right) \subseteq V_{n}^{\mu}$. The initial term $M_{1}$ has already been constructed. Now assume that we have constructed the terms $M_{1}, \ldots, M_{k}, k \geq 1$. To construct $M_{k+1}$ associate with every $n \in \mathbb{N}$ and every $\mu \in M_{k}$ a basis $\left\{V_{n}^{\mu i} \mid i \in \mathbb{N}\right\}$ of open neighborhoods of $u_{n}^{\mu}\left(X_{n}\right)$ in $Z_{n}^{\mu}$. By property (B2) of $\boldsymbol{u}_{n}$, there are indices $\mu_{n}^{i} \geq \mu$ in $M$ such that $r_{n}^{\mu \mu_{n}^{i}}\left(Z_{n}^{\mu_{n}^{i}}\right) \subseteq V_{n}^{\mu i}$. Clearly, $M_{k} \cup\left\{\mu_{n}^{i} \mid(n, i) \in\right.$ $\mathbb{N} \times \mathbb{N}\}$ is a countable subset of $M$. Therefore, by Lemma 5.1, there exists a countable directed subset $M_{k+1} \subseteq M$, which contains $M_{k} \cup\left\{\mu_{n}^{i} \mid(n, i) \in\right.$ $\mathbb{N} \times \mathbb{N}\}$. Now let $n \in \mathbb{N}, \mu \in M_{k}$ and let $V_{n}^{\mu}$ be an open neighborhood of $u_{n}^{\mu}\left(X_{n}\right)$ in $Z_{n}^{\mu}$. There exists an $i \in \mathbb{N}$ such that $V_{n}^{\mu i} \subseteq V_{n}^{\mu}$. Clearly, $\mu^{\prime}=\mu_{n}^{i} \in M_{k+1}, \mu^{\prime} \geq \mu$ and $r_{n}^{\mu \mu^{\prime}}\left(X_{n}^{\mu^{\prime}}\right) \subseteq V_{n}^{\mu i} \subseteq V_{n}^{\mu}$. Clearly, the union $M_{0}=M_{1} \cup M_{2} \cup \ldots$ is a countable directed subset of $M$ such that, for every $n \in \mathbb{N}$, the restrictions $\boldsymbol{u}_{n}\left|M_{0}: X_{n} \rightarrow \boldsymbol{Z}_{n}\right| M_{0}$ have property (B2). Since $M_{0} \supseteq M^{\prime}$, the restrictions $\boldsymbol{u}_{n} \mid M_{0}$ also have property (B1). Consequently, the rectangular system of compact polyhedra $\boldsymbol{Z} \mid\left(\mathbb{N} \times M_{0}\right)$ has the property that the mappings $\boldsymbol{u}_{n}\left|M_{0}: X_{n} \rightarrow \boldsymbol{Z}_{n}\right| M_{0}$ are limits. Since $M_{0}$ is countable, the second statement of Lemma 5.1, applied to $M^{\prime}=M_{0}$, yields an increasing sequence $m_{1} \leq m_{2} \leq \ldots$ of elements of $M_{0}$, which forms a cofinal subset $N_{0}$ of $M_{0}$. Therefore, restricting $\boldsymbol{Z}$ to $\mathbb{N} \times N_{0}$, we obtain a new rectangular system where the mappings $\boldsymbol{u}_{n} \mid N_{0}: X_{n} \rightarrow \boldsymbol{Z}_{n}$ are limits of its vertical systems. If $N_{0}$ is infinite, there is no loss of generality in assuming that $N_{0}=\mathbb{N}$. If $N_{0}$ is finite, we can repeat its last row infinitely many times and thus, again assume that $N_{0}=\mathbb{N}$. 


\section{REFERENCES}

[1] Yu.T. Lisitsa, Second structure theorem for strong homology, Geometric Topology, Dubrovnik 1998, Abstracts, p. 33.

[2] Yu.T. Lisitsa, Basic theorems of strong shape theory of compact Hausdorff spaces, Vestnik Ross. Univ. Druzhby Narodov, Ser. Matematika 2000, No. 6(1), 94-113 (in Russian).

[3] Yu.T. Lisitsa, Second structure theorem for strong homology, Topology Appl. 113 (2001), 107-134.

[4] S. Mardešić, Strong shape and homology, Springer Monographs in Mathematics, Springer, Berlin 2000.

[5] S. Mardešić and J. Segal, Shape theory, North-Holland, Amsterdam 1982.

[6] S. Mardešić and N. Uglešić, On iterated inverse limits, Topology Appl. 120 (2002), $157-167$.

[7] N. Uglešić, Iterated resolutions, Glasnik Mat. 35(55) (2000), 245-259.

[8] T. Watanabe, Approximative expansions of maps into inverse systems, Banach Center Publ., Warsaw, 18 (1986), pp. 363-370.

Department of Mathematics

University of Zagreb

Bijenička cesta 30

10002 Zagreb, P.O. Box 335

Croatia

E-mail: smardes@math.hr

Received: 19.02 .2003

Revised: 10.05 .2003 